\title{
Tidal evolution of close binary asteroid systems
}

\author{
Patrick A. Taylor · Jean-Luc Margot
}

Received: 27 February 2010 / Revised: 12 August 2010 / Accepted: 19 August 2010 /

Published online: 14 September 2010

(C) The Author(s) 2010. This article is published with open access at Springerlink.com

\begin{abstract}
We provide a generalized discussion of tidal evolution to arbitrary order in the expansion of the gravitational potential between two spherical bodies of any mass ratio. To accurately reproduce the tidal evolution of a system at separations less than 5 times the radius of the larger primary component, the tidal potential due to the presence of a smaller secondary component is expanded in terms of Legendre polynomials to arbitrary order rather than truncated at leading order as is typically done in studies of well-separated system like the Earth and Moon. The equations of tidal evolution including tidal torques, the changes in spin rates of the components, and the change in semimajor axis (orbital separation) are then derived for binary asteroid systems with circular and equatorial mutual orbits. Accounting for higher-order terms in the tidal potential serves to speed up the tidal evolution of the system leading to underestimates in the time rates of change of the spin rates, semimajor axis, and mean motion in the mutual orbit if such corrections are ignored. Special attention is given to the effect of close orbits on the calculation of material properties of the components, in terms of the rigidity and tidal dissipation function, based on the tidal evolution of the system. It is found that accurate determinations of the physical parameters of the system, e.g., densities, sizes, and current separation, are typically more important than accounting for higher-order terms in the potential when calculating material properties. In the scope of the long-term tidal evolution of the semimajor axis and the component spin rates, correcting for close orbits is a small effect, but for an instantaneous rate of change in spin rate, semimajor axis, or mean motion, the close-orbit correction can be on the order of tens of percent. This work has possible implications for the determination of the Roche limit and for spin-state alteration during close flybys.
\end{abstract}

Keywords Gravity $\cdot$ Extended body dynamics · Tides · Asteroids · Binary asteroids

P. A. Taylor $(\bowtie)$

Arecibo Observatory, HC 3, Box 53995, Arecibo, PR 00612, USA

e-mail: ptaylor@naic.edu

J.-L. Margot

Departments of Earth \& Space Sciences and Physics \& Astronomy, University of California,

Los Angeles, CA 90095, USA

e-mail: jlm@ess.ucla.edu 


\section{Introduction}

The classic equations for tidal evolution in two-body systems derived or utilized in seminal papers [e.g., MacDonald (1964), Goldreich (1966), Goldreich and Soter (1966), Mignard (1979, 1981)], reviews [e.g., Burns (1977), Weidenschilling et al. (1989), Peale (1999)], and textbooks [e.g., Murray and Dermott (1999), Danby (1992)] are based upon the underlying assumption that the two spherical components in the system are separated by several times the radius of the larger primary component. While this assumption is valid in planet-satellite systems ${ }^{1}$ such as Earth-Moon, Jupiter-Galilean satellites, and Saturn-Titan, as well as for Pluto-Charon and the majority of binary main-belt asteroids (with 100-km-scale primaries), it is not completely accurate for all binary asteroids, especially those in the near-Earth region. Based upon the compilation by Walsh and Richardson (2006) of measured and estimated binary asteroid component size and semimajor axis parameters, nearly $75 \%$ of near-Earth and Mars-crossing binaries have inter-component separations between 3 and 5 primary radii. An updated compilation of parameters by Pravec and Harris (2007) including small main-belt binaries, those with primaries less than $10 \mathrm{~km}$ in diameter, confirms that $75 \%$ of binary systems among these three populations have close mutual orbits. In addition, double asteroids, those systems with equal-size components that were not counted in the above tallies, such as (69230) Hermes (Margot et al. 2003, 2006; Pravec et al. 2003), (90) Antiope (Merline et al. 2000; Michałowski et al. 2004; Descamps et al. 2007), (854) Frostia, (1089) Tama, (1313) Berna, and (4492) Debussy (Behrend et al. 2006), have separations within 5 primary radii. The favored formation mechanism for near-Earth, Mars-crossing, and small main-belt binaries is rotational fission or mass shedding (Margot et al. 2002; Richardson and Walsh 2006; Descamps and Marchis 2008) most likely due to YORP spin-up (Pravec and Harris 2007), a torque on the asteroid spin state due to re-emission of absorbed sunlight (Rubincam 2000; Vokrouhlický and Čapek 2002), where the typical binaries produced have equatorial mutual orbits with semimajor axes between 2 and 4.5 primary radii and eccentricities below 0.15 (Walsh et al. 2008). Though all binary systems in these three populations may not have separations of less than 5 primary radii at present, if formed via spin-up, these systems likely have tidally evolved outward from a closer orbit.

Complex generalized formulae for tidal evolution are presented by Kaula (1964) and Mignard (1980) as extensions of the work of Darwin (1879a,b, 1880) that account for higher-order terms in the expansion of the tidal potential, though, nearly universally, even by Darwin, Kaula, and Mignard themselves, only the leading order is applied in practice under the assumption of a distant secondary and the negligibility of higher-order terms. To date, the most common application of higher-order expansions of the tidal potential is in the Mars-Phobos system where tides on Mars raised by Phobos orbiting at 2.76 Mars radii are causing the gradual infall of Phobos's orbit. As the separation between Mars and Phobos decreases, higher-order terms in the potential expansion must gain importance. With this in mind, attempts to understand the observed secular acceleration of Phobos and the past history of its orbit date back to Redmond and Fish (1964) and have continued with Smith and Born (1976), Lambeck (1979), and Szeto (1983), among others, with Bills et al. (2005) presenting the most recent treatment of the subject.

Because many binaries exist in a regime where traditional assumptions break down, and because tidal evolution is most important at small separations, we are motivated to examine tidal interactions in close orbits. Here, we expand the gravitational potential between two

1 There are small natural satellites of the outer planets that orbit very close to their primaries, but we must keep in mind that these satellites are part of much more complex dynamical systems than simple two-component binaries in addition to having negligible masses compared to their primaries. 
spherical bodies to arbitrary order as well as allow for a secondary of non-negligible mass. We then present the resulting equations for the evolution of the component spin rates and the semimajor axis due to the tidal bulges raised on both components when restricted to systems with mutual orbits that are both circular and equatorial as suggested for small binaries formed via spin-up. The effect of accounting for close orbits is examined and compared to the effect of uncertainties in physical parameters of the binary system.

\section{Tidal potential of arbitrary order}

The potential $V$ per unit mass at a point on the surface of the primary body of mass $M_{\mathrm{p}}$, radius $R_{\mathrm{p}}$, and uniform density $\rho_{\mathrm{p}}$ due to a secondary of mass $M_{\mathrm{s}}$, radius $R_{\mathrm{S}}$, and uniform density $\rho_{\mathrm{S}}$ orbiting on a prograde circular path in the equator plane of the primary with semimajor axis $a$ measured from the center of mass of the primary is

$$
V=-G \frac{M_{\mathrm{s}}}{\Delta}
$$

where $G$ is the gravitational constant and $\Delta$ is the distance between the center of the secondary and the point of interest given by

$$
\Delta=a\left[1-2\left(\frac{R_{\mathrm{p}}}{a}\right) \cos \psi+\left(\frac{R_{\mathrm{p}}}{a}\right)^{2}\right]^{1 / 2},
$$

with $\psi$ measured from the line joining the centers of the primary and secondary [e.g., Murray and Dermott (1999)]. In the spherical polar coordinate system $(r, \theta, \phi)$ shown in Fig. 1, with the polar angle $\theta$ measured from the rotation axis of the primary and the azimuthal angle $\phi$ measured from an arbitrary reference direction fixed in space, the separation angle $\psi$ between the secondary and the point of interest on the primary is

$$
\cos \psi=\cos \theta_{\mathrm{p}} \cos \theta_{\mathrm{s}}+\sin \theta_{\mathrm{p}} \sin \theta_{\mathrm{s}} \cos \left(\phi_{\mathrm{p}}-\phi_{\mathrm{s}}\right) .
$$

For widely separated binary systems where the semimajor axis $a$ is much larger than the radius of the primary $R_{\mathrm{p}}$, the potential is expanded in powers of the small term $R_{\mathrm{p}} / a$ such that

Fig. 1 Geometry for the potential felt on the surface of the primary due to the secondary orbiting a distance $a$ from the center of mass of the primary. The dashed line is the locus of points on the surface of the spherical primary that are separated by the angle $\psi$ and distance $\Delta$ from the position of the spherical secondary and thus feel the same potential

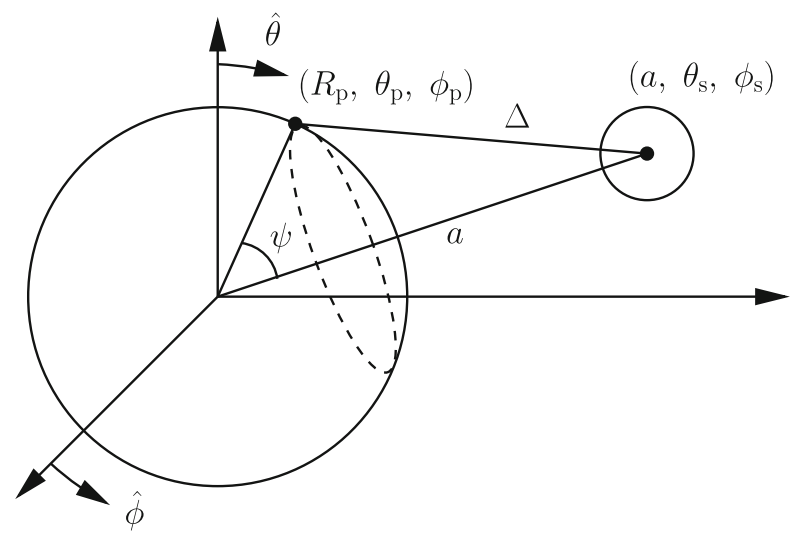




$$
V=-G \frac{M_{\mathrm{s}}}{a}\left[1+\left(\frac{R_{\mathrm{p}}}{a}\right) \cos \psi+\left(\frac{R_{\mathrm{p}}}{a}\right)^{2} \frac{1}{2}\left(3 \cos ^{2} \psi-1\right)+\cdots\right] .
$$

The first term is independent of the position of the point of interest and thus produces no force on the primary. The second term provides the force that keeps the mass element at the point of interest in a circular orbit about the center of mass of the system. The third term is the tidal potential

$$
U=-G \frac{M_{\mathrm{s}} R_{\mathrm{p}}^{2}}{a^{3}} \frac{1}{2}\left(3 \cos ^{2} \psi-1\right)
$$

that is the focus of past studies of tidal evolution where the the bodies are widely separated such as in the Earth-Moon system. However, truncation of the expansion of $V$ in (4) at three terms accurately estimates the true potential in (1) only for separations exceeding $5 R_{\mathrm{p}}$. For smaller separations, as are often found among binary asteroids, higher orders in the expansion of $V$ are necessary.

The full expansion of the potential $V$ in (4) may be written concisely as the sum over Legendre polynomials $P_{\ell}(\cos \psi)$, i.e., zonal harmonic or azimuthally independent surface harmonic functions, as

$$
V=-G \frac{M_{\mathrm{s}}}{a} \sum_{\ell=0}^{\infty}\left(\frac{R_{\mathrm{p}}}{a}\right)^{\ell} P_{\ell}(\cos \psi),
$$

where the $\ell=2$ term of the expansion of $V$ is the dominant tidal term in (5). The full tidal potential $U$ including all orders becomes

$$
U=-G \frac{M_{\mathrm{s}}}{a} \sum_{\ell=2}^{\infty}\left(\frac{R_{\mathrm{p}}}{a}\right)^{\ell} P_{\ell}(\cos \psi) .
$$

While we will derive the tidal evolution equations in terms of an arbitrary order $\ell$, Table 1 lists the order $\ell$ of the expansion necessary for accurate reproduction of the potential $V$ at small separations. At $2 R_{\mathrm{p}}$, the potential must be expanded to at least $\ell=6$, requiring four additional, but manageable, terms in the expansion. This separation is convenient in terms of tidal evolution as it is the contact limit of a binary system with two equal-size components and a reasonable initial separation for the onset of tidal evolution in a newly formed binary

Table 1 Order $\ell$ of Legendre polynomials necessary in the expansion of the gravitational potential (6) of a binary system (with $\psi=0$ ) to accurately reproduce the full potential (1) to within $1 \%$ at separations less than $a / R_{\mathrm{p}} \simeq 5$

\begin{tabular}{lll}
\hline$\ell$ & $a / R_{\mathrm{p}}$ & Legendre polynomial, $\mathrm{P}_{\ell}(\cos \psi)$ \\
\hline 2 & 4.64 & $\frac{1}{4}(1+3 \cos 2 \psi)$ \\
3 & 3.16 & $\frac{1}{8}(3 \cos \psi+5 \cos 3 \psi)$ \\
4 & 2.51 & $\frac{1}{64}(9+20 \cos 2 \psi+35 \cos 4 \psi)$ \\
5 & 2.15 & $\frac{1}{128}(30 \cos \psi+35 \cos 3 \psi+63 \cos 5 \psi)$ \\
6 & 1.93 & $\frac{1}{512}(50+105 \cos 2 \psi+126 \cos 4 \psi+231 \cos 6 \psi)$ \\
\hline
\end{tabular}

If $a / R_{\mathrm{p}}$ is greater than the value listed, expansion to the corresponding order $\ell$ suffices. Recall that the fluid Roche limit is $a / R_{\mathrm{p}}=2.46$ (see Sect. 3). Also note the Legendre polynomials are given in terms of $\cos m \psi$, where $m$ is an integer, rather than the more common form of $\cos ^{m} \psi$ 
system, regardless of component size, especially for systems formed through primary spinup and mass shedding (Walsh et al. 2008). Proceeding inward of $2 R_{\mathrm{p}}$ rapidly requires an unwieldy number of terms in the expansion (e.g., twice as many additional terms are needed at $\left.1.5 R_{\mathrm{p}}\right)$.

\section{Roche limit}

The well-known classical fluid Roche limit is located at $a=2.46 R_{\mathrm{p}}$ (Chandrasekhar 1969) for equal density components, so that if one considers a secondary just outside the fluid limit, one must include the Legendre polynomials of orders $\ell \leq 4$ in the expansion for the potential felt by the primary. For solid, cohesionless ${ }^{2}$ bodies (gravitational aggregates or so-called rubble piles) modeled as a dry soil, the Roche limit falls approximately between $1.5 R_{\mathrm{p}}$ and $2 R_{\mathrm{p}}$ (Holsapple and Michel 2006, 2008; Sharma 2009). The cohesionless Roche limit is based upon a binary system that is not tidally evolving, but the secondary remains stressed by its self-gravity, rotation (synchronized to the orbital period), and the difference in gravity from its near, primary-facing side to its far side. Holsapple and Michel $(2006,2008)$ illustrate that the mass ratio of the components has a negligible effect on the Roche limit, but one would expect that allowing the secondary to have a more rapid spin or allowing for higher-order tidal terms due to its proximity to the primary will increase the internal stresses and push the Roche limit farther from the primary, though, as noted by Sharma (2009), these issues have not been studied in detail.

With a modest amount of cohesion, the secondary may exist within the stated Roche limit (Holsapple and Michel 2008). For the rough properties of a near-Earth binary of $\rho_{\mathrm{p}, \mathrm{s}}=$ $2 \mathrm{~g} / \mathrm{cm}^{3}$ and $R_{\mathrm{S}}=100 \mathrm{~m}$, a cohesion value of $<100 \mathrm{~Pa}$ is enough to hold the secondary together at the surface of the primary. ${ }^{3}$ For comparison, the surface material of comet Tempel 1 excavated by the Deep Impact mission projectile is estimated to have a shear strength of $<65 \mathrm{~Pa}$ (A'Hearn et al. 2005) and an effective strength of $10^{3} \mathrm{~Pa}$ (Richardson et al. 2007); fine-grained terrestrial sand is found to have cohesion values up to $250 \mathrm{~Pa}$ (Schellart 2000). Therefore, it is not unreasonable that in the tidal field of the primary, the secondary can stably exist at the very least within the fluid Roche limit (even if cohesionless), if not also within the cohesionless Roche limit (with a cohesion comparable to comet regolith or sand), justifying our later choice to work to order $\ell=6$ corresponding to a separation of $2 R_{\mathrm{p}}$.

\section{External potential of arbitrary order}

The tidal potential $U_{\ell}$ of arbitrary order $\ell \geq 2$ felt by the primary, taken from (7), may be written concisely as

$$
U_{\ell}=-g_{\mathrm{p}} \zeta_{\ell, \mathrm{p}} P_{\ell}(\cos \psi),
$$

\footnotetext{
2 A cohesionless material has zero shear strength in the absence of confining pressure. The interlocking of the constituent particles under pressure, however, can give the material shear strength.

3 The cohesion needed to prevent disruption scales as the square of both the density and size of the secondary. Thus, for a main-belt binary with a $R_{\mathrm{S}}=10 \mathrm{~km}$, the necessary cohesion is of order $10^{6} \mathrm{~Pa}$, similar to monolithic rock.
} 
where $g_{\mathrm{p}}=G M_{\mathrm{p}} / R_{\mathrm{p}}^{2}$ is the surface gravity of the primary and

$$
\zeta_{\ell, \mathrm{p}}=\frac{M_{\mathrm{s}}}{M_{\mathrm{p}}}\left(\frac{R_{\mathrm{p}}}{a}\right)^{\ell+1} R_{\mathrm{p}} .
$$

The combination $\zeta_{\ell, \mathrm{p}} P_{\ell}(\cos \psi)$ is the equilibrium tide height, due to the tidal potential of order $\ell$, that defines the equipotential surface about a primary that is completely rigid (inflexible). Because the mass ratio $M_{\mathrm{s}} / M_{\mathrm{p}} \leq 1$ and we assume $a \geq 2 R_{\mathrm{p}}$, the quantity $\zeta_{\ell, \mathrm{p}} / R_{\mathrm{p}} \leq 1 / 8$ for all binary systems, and typically $\zeta_{\ell, \mathrm{p}} / R_{\mathrm{p}} \ll 1$.

For a body with realistic rigidity, the tidal potential $U_{\ell}$ physically deforms the surface of the primary by a small distance $\lambda_{\ell, \mathrm{p}} R_{\mathrm{p}} S_{\ell}$ as a function of position on the primary, where $\lambda_{\ell, \mathrm{p}} \ll 1$ and $S_{\ell}$ is a surface harmonic function. Darwin (1879a) and Love (1927) lay the groundwork for showing that, in general, the deformation of a homogeneous density, incompressible sphere

$$
\lambda_{\ell, \mathrm{p}} R_{\mathrm{p}} S_{\ell}=-h_{\ell, \mathrm{p}} \frac{U_{\ell}}{g_{\mathrm{p}}}=h_{\ell, \mathrm{p}} \zeta_{\ell, \mathrm{p}} P_{\ell}(\cos \psi)
$$

is given in terms of the displacement Love number $h_{\ell, \mathrm{p}}$ (Munk and MacDonald 1960),

$$
h_{\ell, \mathrm{p}}=\frac{2 \ell+1}{2(\ell-1)} \frac{1}{1+\frac{\left(2 \ell^{2}+4 \ell+3\right) \mu_{\mathrm{p}}}{\ell g_{\mathrm{p}} \rho_{\mathrm{p}} R_{\mathrm{p}}}},
$$

introducing $\mu_{\mathrm{p}}$ as the rigidity or shear modulus of the primary. ${ }^{4}$ For bodies less than $200 \mathrm{~km}$ in radius, as all components of binary asteroid systems are, the rigidity $\mu$ dominates the stress due to self-gravity $g \rho R \sim G \rho^{2} R^{2}$ (Weidenschilling et al. 1989), even for rubblepile structures [i.e., the model proposed by Goldreich and Sari (2009)], such that the Love number $h_{\ell} \ll 1$ for small bodies. With $h_{\ell, \mathrm{p}}$ and $\zeta_{\ell, \mathrm{p}} / R_{\mathrm{p}}$ small, and noting from (10) that $\lambda_{\ell, \mathrm{p}}=h_{\ell, \mathrm{p}} \zeta_{\ell, \mathrm{p}} / R_{\mathrm{p}}$, the assumption of a small deformation factor $\lambda_{\ell, \mathrm{p}}$ is justified.

Of particular interest is the external potential felt by the secondary now that the primary has been deformed. It is this external potential that produces the tidal torque that transfers angular momentum through the system. Here, we slightly alter our spherical coordinate system such that $\theta$ now measures the angle from the axis of symmetry of the tidal bulge, as in Murray and Dermott (1999), such that the surface of the nearly spherical primary is now given by

$$
R=R_{\mathrm{p}}\left(1+\sum_{\ell=2}^{\infty} \lambda_{\ell, \mathrm{p}} P_{\ell}(\cos \theta)\right) .
$$

The potential felt at a point external to the primary is the sum of the potential of a spherical primary with radius $R_{\mathrm{p}}$ and that of the deformed shell. However, only that due to the deformed shell, called the non-central potential by Murray and Dermott (1999), will contribute to the torque.

In Fig. 2, the reciprocal of the distance $\Delta$ between the external point $(r, \theta, \phi)$ and a point on the surface of the primary $\left(r^{\prime}, \theta^{\prime}, \phi^{\prime}\right)$ separated by an angle $\psi$, where $r^{\prime}=R$ from (12), is

$$
\frac{1}{\Delta}=\frac{1}{r} \sum_{\ell=0}^{\infty}\left(\frac{R_{\mathrm{p}}}{r}\right)^{\ell} P_{\ell}(\cos \psi)+O\left(\lambda_{\ell^{\prime}, \mathrm{p}}\right) .
$$

\footnotetext{
4 Darwin (1879a) realized the correspondence between elastic and viscoelastic media and provides a generalized form for the deformation of a viscous spheroid, a function equivalent to (10) he calls $\sigma$, that when applied to an elastic spheroid, in terms of rigidity rather than viscosity, is equivalent to the expression found here.
} 
Fig. 2 Geometry for the potential felt at an external point due to the deformation of the primary from its initially spherical shape (dashed). Note that here $\theta$ is measured from the axis of symmetry of the tidal bulge

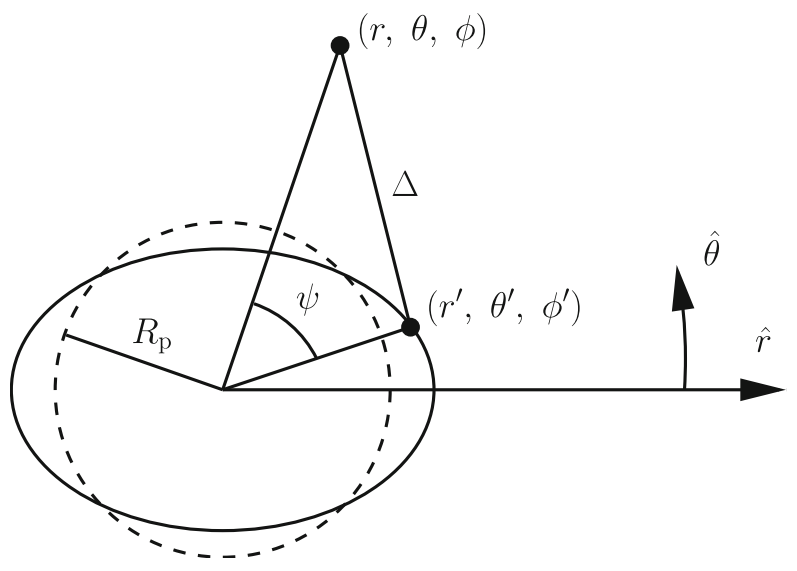

The use of $\ell^{\prime}$ denotes terms based upon the surface deformation rather than the expansion of the distance between the points of interest. The non-central potential (per unit mass of the object disturbed by the potential) due to the deformed shell with mass element $\rho_{\mathrm{p}} R_{\mathrm{p}}^{3} \sum_{\ell^{\prime}=2}^{\infty} \lambda_{\ell^{\prime}, \mathrm{p}} P_{\ell^{\prime}}\left(\cos \theta^{\prime}\right) d\left(\cos \theta^{\prime}\right) d \phi^{\prime}$ is

$U_{\mathrm{nc}}=-G \rho_{\mathrm{p}} R_{\mathrm{p}}^{2}\left(\frac{R_{\mathrm{p}}}{r}\right) \sum_{\ell^{\prime}=2}^{\infty} \sum_{\ell=0}^{\infty} \lambda_{\ell^{\prime}, \mathrm{p}}\left(\frac{R_{\mathrm{p}}}{r}\right)^{\ell} \iint P_{\ell^{\prime}}\left(\cos \theta^{\prime}\right) P_{\ell}(\cos \psi) d\left(\cos \theta^{\prime}\right) d \phi^{\prime}$,

where the double integral goes over the surface of the primary. The integral of the product of two surface harmonics like the Legendre polynomials over a surface is zero unless $\ell=\ell^{\prime}$ such that for a specific order $\ell \geq 2$ (MacRobert 1967),

$$
\begin{aligned}
U_{\ell, \mathrm{nc}} & =-G \rho_{\mathrm{p}} R_{\mathrm{p}}^{2}\left(\frac{R_{\mathrm{p}}}{r}\right) \lambda_{\ell, \mathrm{p}} \times \frac{4 \pi}{2 \ell+1}\left(\frac{R_{\mathrm{p}}}{r}\right)^{\ell} P_{\ell}(\cos \theta) \\
& =-\frac{3}{2 \ell+1} h_{\ell, \mathrm{p}} \zeta_{\ell, \mathrm{p}} g_{\mathrm{p}}\left(\frac{R_{\mathrm{p}}}{r}\right)^{\ell+1} P_{\ell}(\cos \theta) .
\end{aligned}
$$

By defining the more familiar potential Love number

$$
k_{\ell, \mathrm{p}}=\frac{3}{2 \ell+1} h_{\ell, \mathrm{p}}=\frac{3}{2(\ell-1)} \frac{1}{1+\frac{\left(2 \ell^{2}+4 \ell+3\right) \mu_{\mathrm{p}}}{\ell g_{\mathrm{p}} \rho_{\mathrm{p}} R_{\mathrm{p}}}},
$$

which is of a similar order as $h_{\ell, \mathrm{p}}$, the non-central potential is written in the form

$$
U_{\ell, \mathrm{nc}}=-k_{\ell, \mathrm{p}} g_{\mathrm{p}} \zeta_{\ell, \mathrm{p}}\left(\frac{R_{\mathrm{p}}}{r}\right)^{\ell+1} P_{\ell}(\cos \theta)
$$

such that $U_{\ell, \mathrm{nc}}$ at the surface of the primary is simply $k_{\ell, \mathrm{p}} U_{\ell}$. Because $\mu \gg g \rho R$ for small bodies, the Love number $k_{\ell, \mathrm{p}}$ may be approximated by

$$
k_{\ell, \mathrm{p}} \simeq \frac{3}{2(\ell-1)} \frac{\ell}{2 \ell^{2}+4 \ell+3} \frac{g_{\mathrm{p}} \rho_{\mathrm{p}} R_{\mathrm{p}}}{\mu_{\mathrm{p}}}=\frac{2 \pi}{\ell-1} \frac{\ell}{2 \ell^{2}+4 \ell+3} \frac{G \rho_{\mathrm{p}}^{2} R_{\mathrm{p}}^{2}}{\mu_{\mathrm{p}}} .
$$


Taking the external point to be the position of the secondary orbiting at a distance $a$ from the primary, the complete ${ }^{5}$ non-central potential per unit secondary mass due to tides raised on the primary is

$$
\begin{aligned}
U_{\mathrm{nc}} & =-g_{\mathrm{p}} \sum_{\ell=2}^{\infty} k_{\ell, \mathrm{p}} \zeta_{\ell, \mathrm{p}}\left(\frac{a}{R_{\mathrm{p}}}\right)^{-(\ell+1)} P_{\ell}(\cos \theta) \\
& =-\frac{G M_{\mathrm{s}}}{R_{\mathrm{p}}} \sum_{\ell=2}^{\infty} k_{\ell, \mathrm{p}}\left(\frac{a}{R_{\mathrm{p}}}\right)^{-2(\ell+1)} P_{\ell}(\cos \theta) .
\end{aligned}
$$

The non-central potential drops off quickly with increasing separation as the separation to the sixth power for $\ell=2$ and by an additional square of the separation for each successive order. The $\theta$ term in the Legendre polynomial accounts for the angular separation between the external point of interest and the tidal bulge of the primary. For the specific location of the secondary, we define the angle $\delta$ as the geometric lag angle between the axis of symmetry of the tidal bulge and the line connecting the centers of the two components.

\section{Tidal dissipation function $Q$}

In addition to the rigidity $\mu$, the response of a homogeneous, incompressible sphere to a disturbing potential is characterized by the tidal dissipation function $Q$ defined by

$$
Q^{-1}=\frac{1}{2 \pi E^{*}} \oint\left(-\frac{d E}{d t}\right) d t,
$$

where $E^{*}$ is the maximum energy stored in the tidal distortion and the integral is the energy dissipated over one cycle [see Goldreich (1963) or Efroimsky and Williams (2009) for detailed discussions]. This definition is akin to the quality factor in a damped, linear oscillator and does not depend on the details of how the energy is dissipated. Friction in the response of the body to a tide-raising potential plus the rotation of the body itself (at a spin rate $\omega$ compared to the mean motion $n$ in the mutual orbit about the center of mass of the system) lead to misalignment by the geometric lag angle $\delta$.

The geometric lag relates to a phase lag by $\varepsilon_{\ell m p q}=-m \delta \operatorname{sign}(\omega-n)$, where the $\ell m p q$ notation follows Kaula (1964), and we have implicitly assumed a single tidal bulge as done by Gerstenkorn (1955) and MacDonald (1964) by using a single positive geometric lag $\delta$ independent of the tidal frequencies. ${ }^{6}$ The tidal dissipation function $Q$, in turn, relates to the phase angle as $Q_{\ell m p q}^{-1}=\left|\cot \varepsilon_{\ell m p q}\right| \simeq\left|\varepsilon_{\ell m p q}\right|+O\left(\varepsilon_{\ell m p q}^{2}\right)$ (Efroimsky and Williams 2009) provided energy dissipation is weak ( $\left.Q_{\ell m p q} \gg 1\right)$. The absolute value of $\varepsilon_{\ell \mathrm{mpq}}$ is required on physical grounds to ensure that $Q_{\ell m p q}$ is positive. Since the tidal dissipation function

\footnotetext{
5 Here, by complete we mean accounting for all orders $\ell$. We have, however, limited the result to first order in the Love number $k_{\ell, \mathrm{p}}$ because terms of order $\lambda_{\ell, \mathrm{p}}$ were ignored in (13). These would have produced higherorder terms in the Love number in the final form of the potential in (19), but because we have argued $\lambda_{\ell, \mathrm{p}}$ and $k_{\ell, \mathrm{p}}$ are both small quantities, terms of second and higher order in the Love number are negligible.

6 The definition of the phase lag (Kaula 1964; Efroimsky and Williams 2009), when one ignores changes in the periapse and node, is $\varepsilon_{\ell m p q}=[(\ell-2 p+q) n-m \omega] \Delta t_{\ell m p q}$, where the bracketed term is the tidal frequency and $\Delta t_{\ell m p q}$ is the positive time lag in the response of the material to the tidal potential. In the potential expansion by Kaula (1964), only terms satisfying $\ell-2 p=m$ and $q=0$ survive for mutual orbits that are circular and equatorial such that $\varepsilon_{\ell m p q}=-m|\omega-n| \Delta t_{\ell m p q} \operatorname{sign}(\omega-n)=-m \delta \operatorname{sign}(\omega-n)$, assuming a constant time lag and a single (positive) value for geometic lag for all viable combinations of $\ell$ emp.
} 
is related to the phase lag, a different $Q_{\ell m p q}$ technically applies to each tidal frequency. Compared to the dominant order $\ell=2$, where only the $\ell m p q=2200$ term survives in the setup of our problem,

$$
Q_{\ell m p q}^{-1}=m \delta=\frac{m}{2} Q_{2200}^{-1}=\frac{m}{2} Q^{-1}
$$

in general, where we define $Q \equiv Q_{2200}$ such that $Q_{\ell m p q}$ for any tidal frequency is proportional to a single value of $Q$. This simple relation between $Q_{\ell m p q}$ and the $Q$ of the dominant tidal frequency is a direct result of our assumption of a single geometric lag independent of tidal frequency. Such a choice may not be the most realistic physical model, ${ }^{7}$ but does allow for simpler mathematical manipulation. Because $Q$ is necessarily positive regardless of the sign of the phase lag, we append sign $(\omega-n)$ to our forthcoming equations, where the spin rate $\omega$ relates to the tidally distorted component. If $\omega>n$, the bulge leads; if $\omega<n$, the bulge lags behind.

\section{Tidal torques on the components}

The force on the secondary due to the distorted primary at order $\ell$ is $-M_{\mathrm{S}} \nabla U_{\ell, \mathrm{nc}}$, and because we have restricted the problem to a circular, equatorial mutual orbit, the tidal bulge remains in the orbit plane, and the sole component of the force is tangential to the mutual orbit. Returning to the notation where $\psi$ measures the angle from the axis of symmetry of the tidal bulge, ${ }^{8}$ the force at the location of the secondary is proportional to $-\partial P_{\ell} /\left.\partial \psi\right|_{\psi=\delta}$ and pointed in the $-\hat{\psi}$ direction. The value of $\delta$ is taken to be positive as stated in the previous section such that, for $\delta$ small, the quantity $-\partial P_{\ell} /\left.\partial \psi\right|_{\psi=\delta}$ is positive and the primary bulge attracts the secondary. For a prograde mutual orbit with $\omega_{\mathrm{p}}>n$, the primary bulge pulls the secondary ahead in the orbit; if $\omega_{\mathrm{p}}<n$, the primary bulge retards the motion of the secondary (see Fig. 3). The resulting torque vector acting upon the orbit of the secondary, which is located at position $\mathbf{r}$ with respect to the center of mass of the primary, is given by $\Gamma_{\ell, \mathrm{p}}=\mathbf{r} \times\left(-M_{\mathrm{s}} \nabla U_{\ell, \mathrm{nc}}\right)$. Thus, the torque vector, in general, is proportional to $-\partial P_{\ell} /\left.\partial \psi\right|_{\psi=\delta}(\hat{\psi} \times \hat{r})$. As defined, the direction (sign) of $\hat{\psi} \times \hat{r}$ depends on whether the tidal bulge leads or lags, and we indicate this in the magnitude of the torque via the term sign $(\omega-n)$ such that the torque on the orbit of the secondary due to the $\ell$ th-order deformation of the primary is

$$
\begin{aligned}
\Gamma_{\ell, \mathrm{p}} & =-M_{\mathrm{s}} \frac{\partial U_{\ell, \mathrm{nc}}}{\partial \psi_{\mathrm{p}}} \\
& =k_{\ell, \mathrm{p}} \frac{G M_{\mathrm{s}}^{2}}{R_{\mathrm{p}}}\left(\frac{a}{R_{\mathrm{p}}}\right)^{-2(\ell+1)}\left(-\left.\frac{\partial P_{\ell}\left(\cos \psi_{\mathrm{p}}\right)}{\partial \psi_{\mathrm{p}}}\right|_{\psi_{\mathrm{p}}=\delta_{\mathrm{p}}}\right) \operatorname{sign}\left(\omega_{\mathrm{p}}-n\right) .
\end{aligned}
$$

where $\delta_{\mathrm{p}}$ is the geometric lag angle between the primary's tidal bulge and the line of centers. A positive (negative) torque increases (decreases) the energy of the orbit at a rate $\Gamma_{\mathrm{p}} n$. An equal and opposite torque alters the rotational energy of the primary at a rate $-\Gamma_{\mathrm{p}} \omega_{\mathrm{p}}$ such that

\footnotetext{
7 In our model, $Q$ varies inversely with the tidal frequency. Efroimsky and Williams (2009) argue in favor of a rheological model where $Q$ scales to a positive fractional power of the tidal frequency (at least for terrestrial planets). It is unclear what rheological model is proper for gravitational aggregates like binary asteroids.

8 In this notation, the tidal potential in (7) deforms the shape of the component according to (10) and produces the external potential (19) all in terms of the single angle $\psi$.
} 
Fig. 3 When the primary spins faster than the mean motion of the mutual orbit $\left(\omega_{\mathrm{p}}>n\right)$, the tidal bulge is carried ahead of the tide-raising secondary. The resulting torques slow the rotation of the primary and expand the mutual orbit. When the primary spins slower than the mean motion $\left(\omega_{\mathrm{p}}<n\right)$, the torques speed up the rotation of the primary and contract the mutual orbit. Similar diagrams apply to tides raised on the secondary and whether or not $\omega_{\mathrm{s}}>n$. Note that $\psi$ is measured from the axis of symmetry of the tidal bulge of the primary with $\psi=\delta_{\mathrm{p}}$ being the geometric lag angle at the position of the secondary

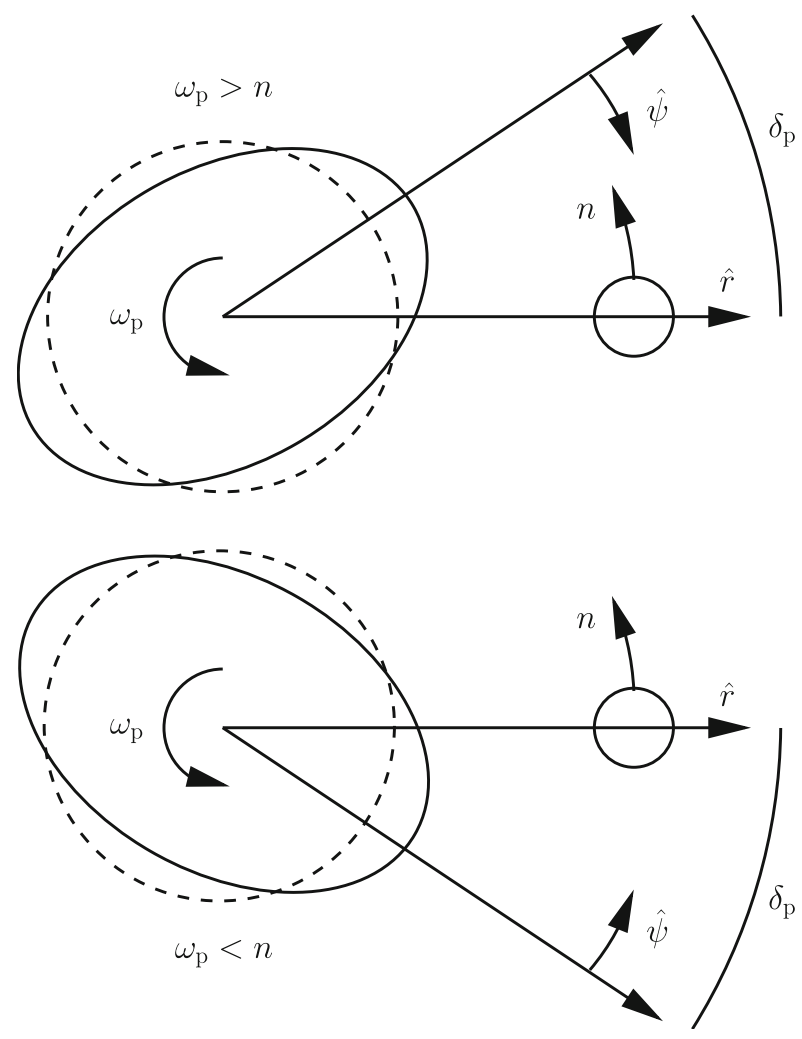

the total energy $E$ of the system is dissipated over time at a rate $\dot{E}=-\Gamma_{\mathrm{p}}\left(\omega_{\mathrm{p}}-n\right)<0$ as heat inside the primary. Though energy is dissipated, angular momentum is conserved due to the equal and opposite nature of the torques on the orbit and the rotation of the primary. Conservation of angular momentum results in the evolution of the mutual orbit and is discussed in the following section.

A similar torque arises from tides raised on the secondary. By the symmetry of motion about the center of mass, the torque $\Gamma_{\ell, \mathrm{s}}$ is given by swapping the subscripts $\mathrm{p}$ and $\mathrm{s}$ in (22) such that

$$
\begin{aligned}
\Gamma_{\ell, \mathrm{s}} & =k_{\ell, \mathrm{s}} \frac{G M_{\mathrm{p}}^{2}}{R_{\mathrm{s}}}\left(\frac{a}{R_{\mathrm{s}}}\right)^{-2(\ell+1)}\left(-\left.\frac{\partial P_{\ell}\left(\cos \psi_{\mathrm{s}}\right)}{\partial \psi_{\mathrm{s}}}\right|_{\psi_{\mathrm{s}}=\delta_{\mathrm{s}}}\right) \operatorname{sign}\left(\omega_{\mathrm{s}}-n\right) \\
& =k_{\ell, \mathrm{p}} \frac{G M_{\mathrm{s}}^{2}}{R_{\mathrm{p}}}\left(\frac{R_{\mathrm{s}}}{R_{\mathrm{p}}}\right)^{2 \ell-3} \frac{\mu_{\mathrm{p}}}{\mu_{\mathrm{s}}}\left(\frac{a}{R_{\mathrm{p}}}\right)^{-2(\ell+1)}\left(-\left.\frac{\partial P_{\ell}\left(\cos \psi_{\mathrm{s}}\right)}{\partial \psi_{\mathrm{s}}}\right|_{\psi_{\mathrm{s}}=\delta_{\mathrm{s}}}\right) \operatorname{sign}\left(\omega_{\mathrm{s}}-n\right),
\end{aligned}
$$

where $\delta_{\mathrm{s}}$ is the geometric lag angle between the tidal bulge of the secondary and the line of centers. This torque changes the orbital energy at a rate $\Gamma_{\mathrm{s}} n$, and the equal and opposite torque alters the rotational energy of the secondary at a rate $-\Gamma_{\mathrm{s}} \omega_{\mathrm{s}}$, dissipating energy as heat in the secondary at a rate $\dot{E}=-\Gamma_{\mathrm{s}}\left(\omega_{\mathrm{s}}-n\right)$. Torques on the primary and secondary weaken for higher orders of $\ell$ and increasing separations, as expected, and do so in the same manner as the non-central potential in (19). Once the rotation rate of a component synchronizes with the mean motion of the mutual orbit, the associated torque goes to zero due to 
the sign $(\omega-n)$ term. ${ }^{9}$ Note that we have ignored interactions between the tidal bulges of the components as these will depend on the square (or higher powers) of the Love numbers, which we have argued are negligible (see Footnote 5).

\section{Spin rate and semimajor axis evolution for close orbits}

During tidal evolution, angular momentum is transferred between the spins of the components and the mutual orbit. For simplicity, assume that the primary and secondary have spin axes parallel to the normal of the mutual orbit plane and rotate in a prograde sense. Then, the torque on the distorted primary alters its spin with time at a rate $\dot{\omega}_{\mathrm{p}}=-\Gamma_{\mathrm{p}} / I_{\mathrm{p}}$, where $I_{\mathrm{p}}=\alpha_{\mathrm{p}} M_{\mathrm{p}} R_{\mathrm{p}}^{2}$ is the moment of inertia of the primary. The pre-factor $\alpha$ is $2 / 5$ for a uniform density sphere, but can vary with the internal structure of the body, and is left as a variable here such that the change in spin rate of the primary is

$$
\dot{\omega}_{\ell, \mathrm{p}}=-\frac{k_{\ell, \mathrm{p}}}{\alpha_{\mathrm{p}}} \frac{\kappa^{2}}{1+\kappa}\left(\frac{a}{R_{\mathrm{p}}}\right)^{-2 \ell+1} n^{2}\left(-\left.\frac{\partial P_{\ell}\left(\cos \psi_{\mathrm{p}}\right)}{\partial \psi_{\mathrm{p}}}\right|_{\psi_{\mathrm{p}}=\delta_{\mathrm{p}}}\right) \operatorname{sign}\left(\omega_{\mathrm{p}}-n\right),
$$

recalling that $-\partial P_{\ell} / \partial \psi \geq 0$ for small angles and defining the mass ratio $\kappa \equiv M_{\mathrm{s}} / M_{\mathrm{p}}=$ $\left(\rho_{\mathrm{S}} / \rho_{\mathrm{p}}\right)\left(R_{\mathrm{S}} / R_{\mathrm{p}}\right)^{3}$. Also note that $n^{2}$, which is proportional to $\left(a / R_{\mathrm{p}}\right)^{-3}$, was introduced via Kepler's Third Law, $n^{2} a^{3}=G\left(M_{\mathrm{p}}+M_{\mathrm{s}}\right)$. For rapidly spinning primaries with $\omega_{\mathrm{p}}>n$, the torque will slow the rotation.

To conserve angular momentum in the system, the change in spin angular momentum, given by the torque $-\Gamma_{\ell, \mathrm{p}}$, plus the change in orbital angular momentum must be zero. The orbital angular momentum for a circular mutual orbit $M_{\mathrm{p}} M_{\mathrm{s}} /\left(M_{\mathrm{p}}+M_{\mathrm{s}}\right) n a^{2}$ changes with time as $(1 / 2) M_{\mathrm{p}} M_{\mathrm{s}} /\left(M_{\mathrm{p}}+M_{\mathrm{s}}\right) n a \dot{a}$ such that conservation requires

$$
\left(\frac{\dot{a}}{R_{\mathrm{p}}}\right)_{\ell, \mathrm{p}}=2 k_{\ell, \mathrm{p}} \kappa\left(\frac{a}{R_{\mathrm{p}}}\right)^{-2 \ell} n\left(-\left.\frac{\partial P_{\ell}\left(\cos \psi_{\mathrm{p}}\right)}{\partial \psi_{\mathrm{p}}}\right|_{\psi_{\mathrm{p}}=\delta_{\mathrm{p}}}\right) \operatorname{sign}\left(\omega_{\mathrm{p}}-n\right)
$$

for each order $\ell$. For rapidly spinning primaries, the orbit will expand as angular momentum is transferred from the spin of the primary to the mutual orbit and, so long as the geometric lag remains small, higher orders will cause both more rapid despinning of the primary and faster expansion of the mutual orbit than $\ell=2$ alone. A large secondary with $\kappa \sim 1$ clearly causes the most rapid tidal evolution. A small secondary with $\kappa \ll 1$ will not cause the primary to despin appreciably due to the $\kappa^{2}$-dependence of (24), but the separation will evolve more readily as (25) scales as $\kappa$.

One can derive the change in the semimajor axis in (25) by other methods including the work done on the orbit and Gauss's formulation of Lagrange's planetary equations. Setting the time derivative of the total energy of the orbit $-G M_{\mathrm{p}} M_{\mathrm{S}} / 2 a$, which is $G M_{\mathrm{p}} M_{\mathrm{s}} \dot{a} / 2 a^{2}$, equal to the work done on the orbit $\Gamma_{\ell, \mathrm{p}} n$ simplifies to (25). Using Gauss's formulation for spherical bodies (see Burns (1976) for a lucid derivation) and a circular mutual orbit,

$$
\dot{a}_{\ell}=\frac{2}{n}(1+\kappa) T_{\ell},
$$

where $T_{\ell}$ is the tangential component of the disturbing force (per unit mass) from the previous section, which is $(1 / a) \partial U_{\ell, \mathrm{nc}} / \partial \psi \operatorname{sign}\left(\omega_{\mathrm{p}}-n\right)$ with $U_{\ell, \mathrm{nc}}$ given by (17). The $(1+\kappa)$

\footnotetext{
9 If the mutual orbit were not circular, a radial tide owing to the eccentricity would continue to act despite the synchronization of the component spin rate to the mean motion.
} 
term is not typically present in the Gauss formulation, but is appended here to the disturbing function ${ }^{10}$ due to the non-inertial nature of the coordinate system centered on the primary (Rubincam 1973) and is necessary, as stated in Darwin (1880), because the primary "must be reduced to rest." One can also argue the term is necessary to account for the reaction of one body to the tidal action of the other (Ferraz-Mello et al. 2008) as the perturbation is internal to the binary system rather than an external element (e.g., drag force, third body). The $(1+\kappa)$ term is absent in the formulae of Kaula (1964), which is reasonable if the secondary is of negligible mass, but we wish to allow for an arbitrary mass ratio. Substitution of the disturbing force into (26) produces (25). By including the $(1+\kappa)$ term in Kaula's equation (38), evaluating Kaula's $F$ and $G$ functions with zero inclination and eccentricity, and recalling that Kaula's $\varepsilon_{\ell m p q}=-m \delta \operatorname{sign}\left(\omega_{\mathrm{p}}-n\right)$ in our notation, we find our evolution of the semimajor axis in (25) is a special case of Kaula's generalization, ${ }^{11}$ as one would expect.

The tidal evolution of the secondary follows similarly. The torque on the distorted secondary alters its spin with time at a rate $\dot{\omega}_{\mathrm{s}}=-\Gamma_{\mathrm{s}} / I_{\mathrm{s}}$,

$$
\begin{aligned}
\dot{\omega}_{\ell, \mathrm{s}} & =-\frac{k_{\ell, \mathrm{s}}}{\alpha_{\mathrm{s}}} \frac{1}{\kappa(1+\kappa)}\left(\frac{R_{\mathrm{S}}}{R_{\mathrm{p}}}\right)^{2 \ell-1}\left(\frac{a}{R_{\mathrm{p}}}\right)^{-2 \ell+1} n^{2}\left(-\left.\frac{\partial P_{\ell}\left(\cos \psi_{\mathrm{s}}\right)}{\partial \psi_{\mathrm{s}}}\right|_{\psi_{\mathrm{s}}=\delta_{\mathrm{s}}}\right) \operatorname{sign}\left(\omega_{\mathrm{s}}-n\right) \\
& =-\frac{k_{\ell, \mathrm{p}}}{\alpha_{\mathrm{p}}} \frac{\kappa}{1+\kappa}\left(\frac{R_{\mathrm{s}}}{R_{\mathrm{p}}}\right)^{2 \ell-5} \frac{\alpha_{\mathrm{p}}}{\alpha_{\mathrm{s}}} \frac{\mu_{\mathrm{p}}}{\mu_{\mathrm{s}}}\left(\frac{a}{R_{\mathrm{p}}}\right)^{-2 \ell+1} n^{2}\left(-\left.\frac{\partial P_{\ell}\left(\cos \psi_{\mathrm{s}}\right)}{\partial \psi_{\mathrm{s}}}\right|_{\psi_{\mathrm{s}}=\delta_{\mathrm{s}}}\right) \operatorname{sign}\left(\omega_{\mathrm{s}}-n\right),
\end{aligned}
$$

and alters the semimajor axis of the mutual orbit at a rate of

$$
\left(\frac{\dot{a}}{R_{\mathrm{p}}}\right)_{\ell, \mathrm{s}}=2 k_{\ell, \mathrm{p}} \kappa\left(\frac{R_{\mathrm{s}}}{R_{\mathrm{p}}}\right)^{2 \ell-3} \frac{\mu_{\mathrm{p}}}{\mu_{\mathrm{s}}}\left(\frac{a}{R_{\mathrm{p}}}\right)^{-2 \ell} n\left(-\left.\frac{\partial P_{\ell}\left(\cos \psi_{\mathrm{s}}\right)}{\partial \psi_{\mathrm{s}}}\right|_{\psi_{\mathrm{s}}=\delta_{\mathrm{s}}}\right) \operatorname{sign}\left(\omega_{\mathrm{s}}-n\right) .
$$

The Legendre polynomials in Table 1 are written as sums of terms of the form cos $m \psi$ where $m$ is an integer (see Footnote 11). Thus, the derivative $\partial P_{\ell} /\left.\partial \psi\right|_{\psi=\delta}$ is a sum of terms of the form $\sin m \delta$. For small geometric lag angles $(Q \gg 1),-\partial P_{\ell} /\left.\partial \psi\right|_{\psi=\delta} \geq 0$ and $\sin m \delta \simeq m \delta$ such that $-\partial P_{\ell} /\left.\partial \psi\right|_{\psi=\delta} \propto Q^{-1}$. Because the derivative of a Legendre polynomial is proportional to $Q^{-1}$, only the size ratio of the components and their material properties, in terms of their respective $\mu Q$ values, determine the relative strength of the torques and the relative contributions to the orbit expansion,

$$
\left|\frac{\Gamma_{\ell, \mathrm{s}}}{\Gamma_{\ell, \mathrm{p}}}\right|=\left|\frac{\dot{a}_{\ell, \mathrm{s}}}{\dot{a}_{\ell, \mathrm{p}}}\right|=\left(\frac{R_{\mathrm{s}}}{R_{\mathrm{p}}}\right)^{2 \ell-3} \frac{\mu_{\mathrm{p}} Q_{\mathrm{p}}}{\mu_{\mathrm{s}} Q_{\mathrm{s}}},
$$

\footnotetext{
${ }^{10}$ Algebraically, from the time rate of change of the orbital energy, $\dot{a}=2 a^{2} \dot{E} / G M_{\mathrm{p}} M_{\mathrm{S}}$, and the change in orbital energy is further related to the velocity of the secondary $\dot{\mathbf{r}}$ and the disturbing force $\mathbf{F}=-M_{\mathrm{S}} \nabla U_{\mathrm{nc}}$ such that $\dot{E}=\dot{\mathbf{r}} \cdot \mathbf{F}=n a M_{\mathrm{S}} T$ for a circular mutual orbit. Replacing $\dot{E}$ by $n a M_{\mathrm{S}} T$ and using Kepler's Third Law, $n^{2} a^{3}=G\left(M_{\mathrm{p}}+M_{\mathrm{s}}\right)=G M_{\mathrm{p}}(1+\kappa)$, in the expression for $\dot{a}$ gives (26) for a specific order $\ell$. If $M_{\mathrm{S}}$ were ignored in Kepler's Third Law, the more familiar form of Gauss's formulation would emerge: $\dot{a}=2 T / n$. 11 The product of Kaula's $F_{\ell m p}$ and $G_{\ell p q}$ functions is non-zero for circular, equatorial orbits only if $\ell-2 p=$ $m$ and $q=0$. The prefactors on each $\psi$ in the Legendre polynomials listed in Table 1 are the values of $m$ for each order $\ell$ that satisfy $\ell-2 p=m$. Thus, the cosine terms in the Legendre polynomials we list correspond to $\ell m p q$ of $2200,3110,3300,4210,4400,5120,5310,5500,6220,6410$, and 6600. This correspondence allows us to link our equations written in terms of Legendre polynomials and a geometric lag to Kaula's equations written in terms of the phase lag $\varepsilon_{\ell m p q}$. While the combinations 2010, 4020, and 6030 satisfy $\ell-2 p=m$, terms with $m=0$ cannot contribute to the tidal evolution of the system because, by definition, these terms do not produce a phase lag. These three terms are responsible for the $\psi$-independent components of the Legendre polynomials with $\ell=2,4,6$ that vanish upon differentiation with respect to $\psi$.
} 
with the relative contribution of the secondary decreasing at higher orders of $\ell$ and for smaller secondaries. Note that the relative strength of the torques is independent of the mass and density. ${ }^{12}$ For classical $\ell=2$ tides on components with similar material properties, the torque due to the distorted secondary is a factor of the size ratio weaker than the torque due to the distorted primary. For each higher order in the expansion, the relative strength of the torque due to the distorted secondary weakens by the square of the size ratio. The changes in the spin rates compare as

$$
\left|\frac{\dot{\omega}_{\ell, \mathrm{s}}}{\dot{\omega}_{\ell, \mathrm{p}}}\right|=\frac{1}{\kappa}\left(\frac{R_{\mathrm{s}}}{R_{\mathrm{p}}}\right)^{2 \ell-5} \frac{\alpha_{\mathrm{p}}}{\alpha_{\mathrm{s}}} \frac{\mu_{\mathrm{p}} Q_{\mathrm{p}}}{\mu_{\mathrm{s}} Q_{\mathrm{s}}}=\frac{\rho_{\mathrm{p}}}{\rho_{\mathrm{s}}}\left(\frac{R_{\mathrm{s}}}{R_{\mathrm{p}}}\right)^{2(\ell-4)} \frac{\alpha_{\mathrm{p}}}{\alpha_{\mathrm{s}}} \frac{\mu_{\mathrm{p}} Q_{\mathrm{p}}}{\mu_{\mathrm{s}} Q_{\mathrm{s}}} .
$$

This differs from a generalization of Darwin's result (c.f. Darwin (1879b), p. 521) because we have included the ratio of the Love numbers of the components. At the dominant orders, $\ell=2$ and 3 , with similar densities, shapes, and material properties, the spin rate of the secondary changes faster than the primary. However, interestingly, for $\ell=4$, the contributions to the changes in spin rates are equal, and for orders $\ell>4$, the contribution to the change in spin rate of the primary is greater than that of the secondary. As with the torques, the relative strength of the changes in spin rates weakens by the square of the size ratio for each successive order $\ell$. For smaller secondaries, the changes in spin rates are smaller than for similar mass components, and, for all cases, the process of changing the spin of the primary is slower than for the secondary.

Evaluating the Love number $k_{\ell, \mathrm{p}}$ in (18) and $\partial P_{\ell} / \partial \psi_{\mathrm{p}}$ from Table 1 explicitly for orders $\ell \leq 6$, assuming a small geometric lag angle $\delta_{\mathrm{p}}$, and applying (21), the spin of the primary changes as

$$
\begin{aligned}
\dot{\omega}_{\mathrm{p}}= & -\frac{8}{19} \frac{1}{\alpha_{\mathrm{p}}} \frac{\pi^{2} G^{2} \rho_{\mathrm{p}}^{3} R_{\mathrm{p}}^{2}}{\mu_{\mathrm{p}} Q_{\mathrm{p}}} \kappa^{2}\left(\frac{a}{R_{\mathrm{p}}}\right)^{-6} \operatorname{sign}\left(\omega_{\mathrm{p}}-n\right) \\
& \times\left[1+\frac{19}{22}\left(\frac{a}{R_{\mathrm{p}}}\right)^{-2}+\frac{380}{459}\left(\frac{a}{R_{\mathrm{p}}}\right)^{-4}+\frac{475}{584}\left(\frac{a}{R_{\mathrm{p}}}\right)^{-6}+\frac{133}{165}\left(\frac{a}{R_{\mathrm{p}}}\right)^{-8}\right],
\end{aligned}
$$

where $n$ has been replaced with Kepler's Third Law to show the full dependence upon the separation of the components $a / R_{\mathrm{p}}$. Using either (27) or (30), the spin of the secondary changes as

$$
\begin{aligned}
\dot{\omega}_{\mathrm{s}}= & -\frac{8}{19} \frac{1}{\alpha_{\mathrm{s}}} \frac{\pi^{2} G^{2} \rho_{\mathrm{p}}^{3} R_{\mathrm{p}}^{2}}{\mu_{\mathrm{s}} Q_{\mathrm{s}}} \kappa\left(\frac{R_{\mathrm{s}}}{R_{\mathrm{p}}}\right)^{-1}\left(\frac{a}{R_{\mathrm{p}}}\right)^{-6} \operatorname{sign}\left(\omega_{\mathrm{s}}-n\right) \\
\times & {\left[1+\frac{19}{22}\left(\frac{R_{\mathrm{S}}}{R_{\mathrm{p}}}\right)^{2}\left(\frac{a}{R_{\mathrm{p}}}\right)^{-2}+\frac{380}{459}\left(\frac{R_{\mathrm{S}}}{R_{\mathrm{p}}}\right)^{4}\left(\frac{a}{R_{\mathrm{p}}}\right)^{-4}\right.} \\
& \left.+\frac{475}{584}\left(\frac{R_{\mathrm{S}}}{R_{\mathrm{p}}}\right)^{6}\left(\frac{a}{R_{\mathrm{p}}}\right)^{-6}+\frac{133}{165}\left(\frac{R_{\mathrm{s}}}{R_{\mathrm{p}}}\right)^{8}\left(\frac{a}{R_{\mathrm{p}}}\right)^{-8}\right] .
\end{aligned}
$$

Assuming similar densities for the components, the change in the spin rate of the primary scales as the size ratio of the components to the sixth power $\left(\propto \kappa^{2}\right)$; the spin rate of the secondary scales only as the square of the size ratio at leading order, reinforcing from (30) how the spin of the secondary evolves more rapidly than that of the primary, especially for small size ratios.

12 However, the absolute strengths of the torques in (22) and (23) do depend on the masses and densities of the components. 
For close orbits, the separation of the components changes as angular momentum is transferred to or from the spins of the components such that the overall change in the orbital separation for $\ell \leq 6$ is the sum of (25) and (28),

$$
\begin{aligned}
\frac{\dot{a}}{R_{\mathrm{p}}}= & \frac{8 \sqrt{3}}{19} \frac{\pi^{3 / 2} G^{3 / 2} \rho_{\mathrm{p}}^{5 / 2} R_{\mathrm{p}}^{2}}{\mu_{\mathrm{p}} Q_{\mathrm{p}}} \kappa(1+\kappa)^{1 / 2}\left(\frac{a}{R_{\mathrm{p}}}\right)^{-11 / 2} \\
& \times\left[\operatorname{sign}\left(\omega_{\mathrm{p}}-n\right)+\left(\frac{R_{\mathrm{s}}}{R_{\mathrm{p}}}\right) \frac{\mu_{\mathrm{p}} Q_{\mathrm{p}}}{\mu_{\mathrm{s}} Q_{\mathrm{s}}} \operatorname{sign}\left(\omega_{\mathrm{s}}-n\right)\right. \\
& +\frac{19}{22}\left(\frac{a}{R_{\mathrm{p}}}\right)^{-2}\left(\operatorname{sign}\left(\omega_{\mathrm{p}}-n\right)+\left(\frac{R_{\mathrm{s}}}{R_{\mathrm{p}}}\right)^{3} \frac{\mu_{\mathrm{p}} Q_{\mathrm{p}}}{\mu_{\mathrm{s}} Q_{\mathrm{s}}} \operatorname{sign}\left(\omega_{\mathrm{s}}-n\right)\right) \\
& +\frac{380}{459}\left(\frac{a}{R_{\mathrm{p}}}\right)^{-4}\left(\operatorname{sign}\left(\omega_{\mathrm{p}}-n\right)+\left(\frac{R_{\mathrm{s}}}{R_{\mathrm{p}}}\right)^{5} \frac{\mu_{\mathrm{p}} Q_{\mathrm{p}}}{\mu_{\mathrm{s}} Q_{\mathrm{s}}} \operatorname{sign}\left(\omega_{\mathrm{s}}-n\right)\right) \\
& +\frac{475}{584}\left(\frac{a}{R_{\mathrm{p}}}\right)^{-6}\left(\operatorname{sign}\left(\omega_{\mathrm{p}}-n\right)+\left(\frac{R_{\mathrm{s}}}{R_{\mathrm{p}}}\right)^{7} \frac{\mu_{\mathrm{p}} Q_{\mathrm{p}}}{\mu_{\mathrm{s}} Q_{\mathrm{s}}} \operatorname{sign}\left(\omega_{\mathrm{s}}-n\right)\right) \\
& \left.+\frac{133}{165}\left(\frac{a}{R_{\mathrm{p}}}\right)^{-8}\left(\operatorname{sign}\left(\omega_{\mathrm{p}}-n\right)+\left(\frac{R_{\mathrm{s}}}{R_{\mathrm{p}}}\right)^{9} \frac{\mu_{\mathrm{p}} Q_{\mathrm{p}}}{\mu_{\mathrm{s}} Q_{\mathrm{s}}} \operatorname{sign}\left(\omega_{\mathrm{s}}-n\right)\right)\right] .
\end{aligned}
$$

Inside the square brackets, having a secondary of negligible size $\left(R_{\mathrm{S}} / R_{\mathrm{p}} \rightarrow 0\right)$ has the same effect as having a synchronous secondary $\left(\omega_{\mathrm{s}}=n\right)$; both make the contribution from the secondary vanish. Of course, if one considers the factor outside the square brackets, having a secondary of negligible size makes the mass ratio $\kappa$ negligible, while having a synchronous secondary does not directly affect $\kappa$. The change in the mean motion of the mutual orbit follows from Kepler's Third Law and (33) as

$$
\frac{\dot{n}}{n}=-\frac{3}{2}\left(\frac{a}{R_{\mathrm{p}}}\right)^{-1}\left(\frac{\dot{a}}{R_{\mathrm{p}}}\right) .
$$

Note that in the above Eqs. (31-34), any difference in density between the components is accounted for in the mass ratio $\kappa$; otherwise, only the size ratio of the components is involved in the terms due to tides raised on the secondary. Obviously, the contribution of the secondary is most important when the components are of similar size. Not only is the contribution of the secondary weakened because of its smaller size, it should also be despun faster than the primary such that its contribution turns off when $\omega_{\mathrm{s}}=n$ long before the primary does the same. Furthermore, each equation has a strong inverse dependence on the separation of the components even at $\ell=2$, and while the inclusion of higher-order terms will be strongest at small separations, the orbit of a typical outwardly evolving system will expand to a wider separation rapidly.

\section{Effect of close orbit expansion on tidal evolution}

Inclusion of higher-order terms for the changes in spin rates and semimajor axis in (31-33) speeds up the evolution of the system and decreases the tidal timescales. Using up to order $\ell=6$ compared to $\ell=2$ results in the spin rates of the components changing up to $28 \%$ faster at $2 R_{\mathrm{p}}$, but falling off quickly with increasing separation (Fig. 4 ) to less than $4 \%$ at $5 R_{\mathrm{p}}$. The size ratio of the components only affects $\dot{\omega}_{\mathrm{s}}$, where the higher-order terms are weaker 
Fig. 4 Time rates of change of the spin rate of the primary (left) and secondary (right) as a function of the separation of the components using all orders up to $\ell=6$ versus using classical $\ell=2$ tides only. The plotted ratio amounts to the bracketed portions of (31) and (32) for the primary and secondary, respectively. The change in spin rate of the primary due to higher-order terms is unaffected by the size ratio of the components. The change in spin rate of the secondary is greater for larger size ratios, plotted from top to bottom with $R_{\mathrm{S}} / R_{\mathrm{p}}=1,0.8,0.6,0.4$, and 0.2 . The effect of higher-order terms is always below $30 \%$ beyond $2 R_{\mathrm{p}}$ and falls below $1 \%$ by a separation of $10 R_{\mathrm{p}}$ for all size ratios
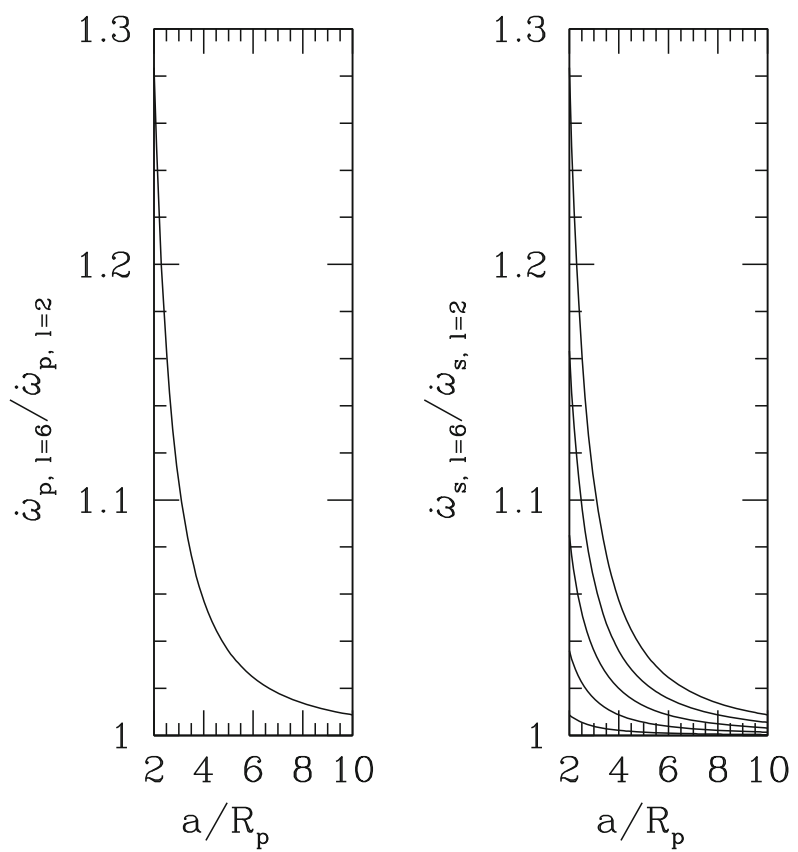

for smaller secondaries. Similarly, for the change in semimajor axis with time, assuming both components are causing the separation to change in the same sense (sign $\left(\omega_{\mathrm{p}}-n\right)$ and $\operatorname{sign}\left(\omega_{\mathrm{s}}-n\right)$ have the same value), using up to order $\ell=6$ (Fig. 5) results in a faster evolution by $21-28 \%$ at $2 R_{\mathrm{p}}$ and decreases quickly with increasing separation. Unlike the changes in spin rates, the largest effect on the evolution of the semimajor axis occurs when the size ratio is either unity (equal size) or negligible or when the spin of the secondary has synchronized to the mean motion such that the tidal torque on the secondary vanishes. The change in semimajor axis with time is least affected by the higher-order terms for a size ratio of 0.53 with all other size ratios falling within these bounds. According to (34) for the change in the mean motion with time, the value of $\dot{n} / n$ using higher-order terms compared to $\ell=2$ has the same form as the change in semimajor axis in Fig. 5.

The strengths of the contributions of the extra terms in the close-orbit correction to the change in semimajor axis are listed in Table 2 . At $2 R_{\mathrm{p}}$, higher-order terms with $\ell \geq 3$ account for nearly $25 \%$ of the change in semimajor axis with time. Although the $\ell=6$ term is necessary for accurate reproduction of the potential between the bodies to within $1 \%$ at $2 R_{\mathrm{p}}$, it does not alter the change in semimajor axis with time at the $1 \%$ level because of the stronger dependence of (25) on separation compared to (6). The net contribution of the higher-order terms in Table 2 decreases by roughly $5 \%$ at each value of the separation from Table 1 with only the $\ell=3$ term having much consequence beyond $3 R_{\mathrm{p}}$.

The total change in the component spin rates as a function of separation, shown in Fig. 6, is given by integration of the ratio of (31) and (33) for the primary and the ratio of (32) and (33) for the secondary. Depending on the size ratio of the components, the total change in the spin rate of the primary is enhanced by up to $6 \%$ at $2 R_{\mathrm{p}}$ over using $\ell=2$ tides only, but not by more than a few percent at larger separations. For the secondary, perhaps counter-intuitively, despite the spin of the secondary evolving more rapidly with time by adding higher-order terms (Fig. 4), its evolution with respect to the separation is less than when using $\ell=2$ only; 


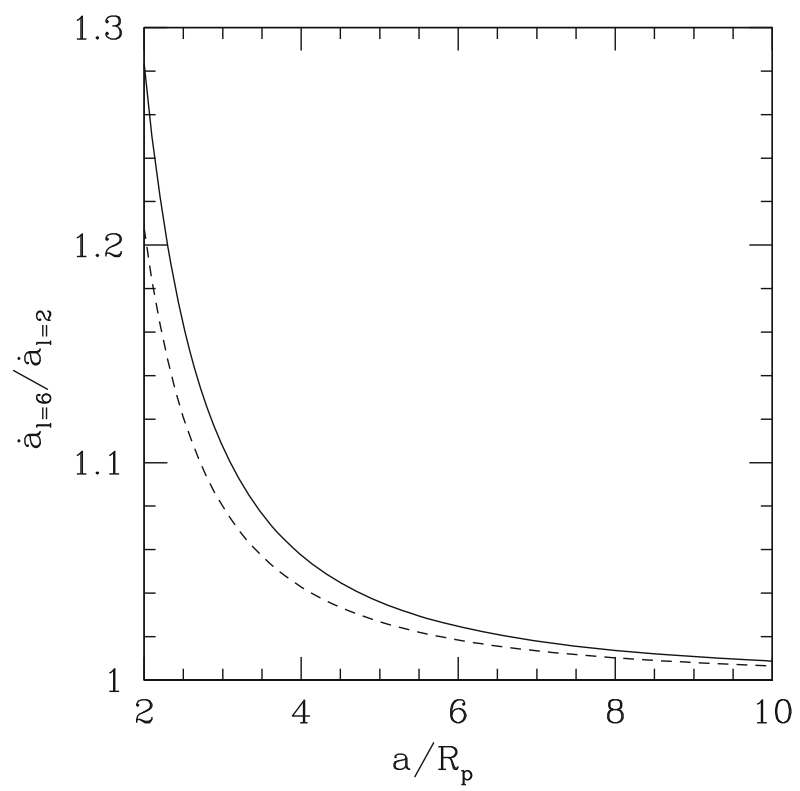

Fig. 5 Time rate of change of the semimajor axis of the mutual orbit as a function of the separation of the components using all orders up to $\ell=6$ versus using classical $\ell=2$ tides. The plotted ratio amounts to the bracketed portion of (33) divided by $1+R_{\mathrm{S}} / R_{\mathrm{p}}$ with both components having similar $\mu Q$ parameters and contributing to the evolution in an additive sense. The solid curve corresponds to a system with components of equal size $\left(R_{\mathrm{S}} / R_{\mathrm{p}}=1\right)$, a secondary of negligible size $\left(R_{\mathrm{S}} / R_{\mathrm{p}}=0\right)$, or a synchronized secondary $\left(\omega_{\mathrm{S}}=n\right)$. The lower bound (dashed curve) is for the size ratio $R_{\mathrm{S}} / R_{\mathrm{p}}=0.53$. As in Fig. 4 , the effect of higher-order terms is always below $30 \%$ beyond $2 R_{\mathrm{p}}$ and falls below $1 \%$ by a separation of $10 R_{\mathrm{p}}$ for all size ratios

Table 2 Maximum contributions in percentages by the successive orders $\ell$ that alter the semimajor axis of the mutual orbit in (33) at the separations listed in Table 1

\begin{tabular}{lccccc}
\hline$\dot{a}_{\ell} / \dot{a}$ & $a / R_{\mathrm{p}}=1.93$ & $a / R_{\mathrm{p}}=2.15$ & $a / R_{\mathrm{p}}=2.51$ & $a / R_{\mathrm{p}}=3.16$ & $a / R_{\mathrm{p}}=4.64$ \\
\hline$\dot{a}_{2} / \dot{a}$ & 76.25 & 80.93 & 86.08 & 91.27 & 95.97 \\
$\dot{a}_{3} / \dot{a}$ & 17.68 & 15.12 & 11.80 & 7.89 & 3.85 \\
$\dot{a}_{4} / \dot{a}$ & 4.55 & 3.14 & 1.80 & 0.76 & 0.17 \\
$\dot{a}_{5} / \dot{a}$ & 1.20 & 0.67 & 0.28 & 0.07 & 0.01 \\
$\dot{a}_{6} / \dot{a}$ & 0.32 & 0.14 & 0.04 & 0.01 & -
\end{tabular}

The strengths of the contributions depend on the size ratio of the components with systems having negligibly small secondaries or equal-size components having the strongest contributions from higher order terms, which are shown here. Having a synchronous secondary $\left(\omega_{\mathrm{s}}=n\right)$ also has the same effect on $\dot{a}_{\ell} / \dot{a}$. It is assumed the components have similar $\mu Q$ parameters and the effect of each component's tides on the semimajor axis are additive

the deficit is as large as $22 \%$ at $2 R_{\mathrm{p}}$ when the size of the secondary is negligible. This is because for smaller secondaries, the effect of higher-order terms on $\dot{\omega}_{\mathrm{s}}$ in (32) is reduced, while the effect of higher-order terms on $\dot{\omega}_{\mathrm{p}}$ is independent of the size ratio. Thus, for a rapidly rotating primary, the higher-order terms transfer more angular momentum from the spin of the primary to the orbit, expanding the separation faster than by $\ell=2$ tides alone and faster than the spin rate of the secondary changes such that the net effect on $\Delta \omega_{\mathrm{s}}(a)$ is smaller. 


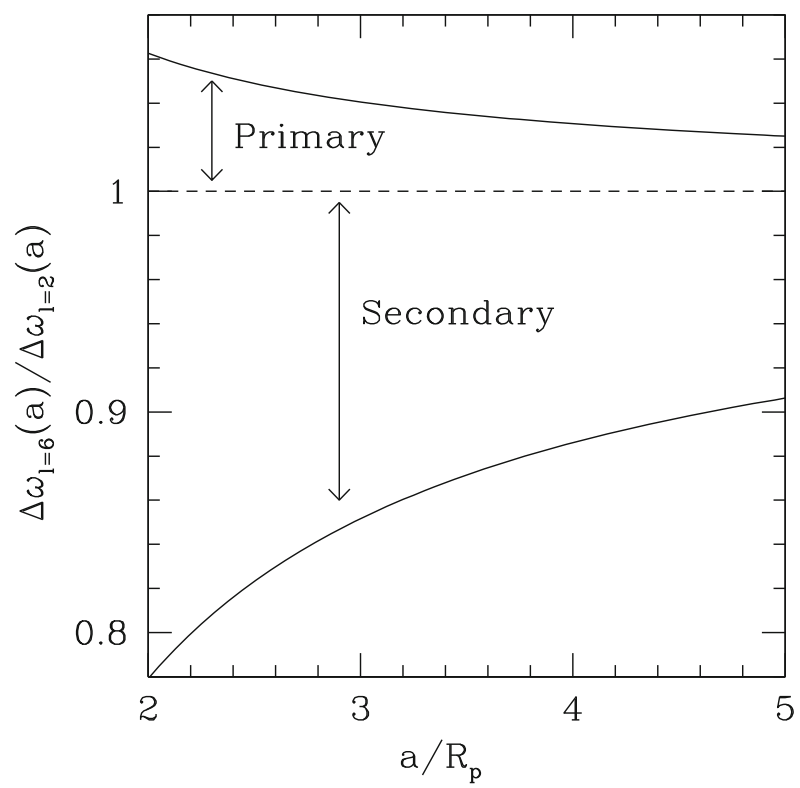

Fig. 6 Total change in spin rate of the components based on tidal evolution from an initial separation of $2 R_{\mathrm{p}}$ using all orders up to $\ell=6$ versus using classical $\ell=2$ tides. The coordinate on the $x$-axis is the final separation of the tidal evolution. With both components contributing in an additive sense in (33), the spin rate of the primary is affected more rapidly than in the classical case, while the secondary is affected less rapidly. The maximum change in the spin rate of the primary occurs for $R_{\mathrm{S}} / R_{\mathrm{p}}=0.53$ (upper solid curve) and the minimum is the dashed line at 1 for $R_{\mathrm{S}} / R_{\mathrm{p}}=0,1$ or a synchronized secondary. The lower solid curve corresponds to the change in spin rate of the secondary for $R_{\mathrm{S}} / R_{\mathrm{p}} \rightarrow 0$. For larger size ratios, the curve for the secondary moves toward the dashed line at 1

Fig. 7 Evolution of the semimajor axis with time using all orders up to $\ell=6$ versus using classical $\ell=2$ tides. Time is plotted logarithmically and scaled to the time necessary for a system to evolve from $2 R_{\mathrm{p}}$ to $5 R_{\mathrm{p}}$ via $\ell=2$ tides. As in Fig. 5 , the solid curve corresponds to $R_{\mathrm{S}} / R_{\mathrm{p}}=0,1$ or a synchronized secondary and the dashed curve corresponds to $R_{\mathrm{S}} / R_{\mathrm{p}}=0.53$. Using up to $\ell=6$ gives a correction of order $1 \%$ to classical tides at any point in the evolution from $2 R_{\mathrm{p}}$ to $5 R_{\mathrm{p}}$

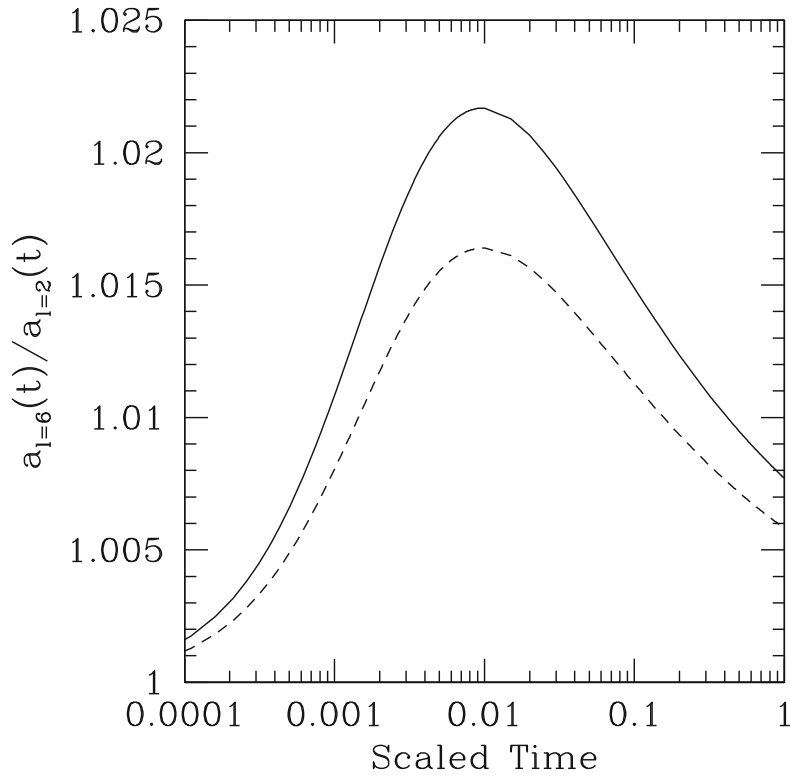


Integration of (33) provides the separation as a function of time. For tidal evolution from an initial separation of $2 R_{\mathrm{p}}$ to a final separation of $5 R_{\mathrm{p}}$ (Fig. 7), the close-orbit correction is strongest at the onset, expanding the separation more rapidly than $\ell=2$ tides, but only by about $2 \%$ over the same time interval. The contributions from the higher-order terms lose strength over time as the separation increases resulting in a net effect of expanding the separation by $\sim 1 \%$ extra by using $\ell=6$ instead of $\ell=2$. From Figs. 6 and 7 , the integrated effects of the close-orbit correction are small, typically of order a few percent; the effects are more noticeable in the instantaneous rates of change of the spin rates, separation, or mean motion due to the rapid fall-off in strength of the higher-order terms with increasing separation and how rapidly the system tidally evolves from small separations.

Rearrangement and integration of (33) allows one to calculate the combination of the material properties of the components $\mu Q$ (assuming $\mu_{\mathrm{p}} Q_{\mathrm{p}}=\mu_{\mathrm{s}} Q_{\mathrm{s}}$ ) and the age of the binary $\Delta t$ based on measurable system parameters. For brevity, we retain only terms due to tides raised on the primary giving

$$
\begin{aligned}
\frac{\mu Q}{\Delta t}= & \frac{8 \sqrt{3}}{19} \pi^{3 / 2} G^{3 / 2} \rho_{\mathrm{p}}^{5 / 2} R_{\mathrm{p}}^{2} \kappa(1+\kappa)^{1 / 2} \\
& \times\left[\int_{2}^{a_{\mathrm{f}} / R_{\mathrm{p}}} \frac{x^{11 / 2}}{1+\frac{19}{22} x^{-2}+\frac{380}{459} x^{-4}+\frac{475}{584} x^{-6}+\frac{133}{165} x^{-8}} \mathrm{~d} x\right]^{-1}
\end{aligned}
$$

with $x=a / R_{\mathrm{p}}$. Because both terms on the left-hand side of (35) are unknown, one may either estimate the material properties by assuming binary ages (Margot et al. 2002, 2003; Taylor and Margot 2007), estimate binary ages by assuming material properties (Walsh and Richardson 2006; Goldreich and Sari 2009), or consider both avenues (Marchis et al. 2008b,a; Taylor and Margot 2010). Furthermore, precisely because both terms are unknown, assuming a value for one has an intimate effect on the calculation of the other as changing one's value by an order of magnitude changes the result of the other by an order of magnitude. Thus, when one wishes to find $\mu Q$, for instance, choosing an age for the binary injects a great source of uncertainty into the calculation.

The close-orbit correction enhances the rate at which the separation changes such that, to provide the same tidal evolution over the same timescale $\Delta t$, the product $\mu Q$ must increase to compensate for the inclusion of the higher-order terms. For classical $\ell=2$ tides, the denominator of the integrand in (35) vanishes such that the effect of including terms up to $\ell=6$ alters $\mu Q$ according to

$$
\frac{\mu Q_{\ell=6}}{\mu Q_{\ell=2}}=\frac{\int_{2}^{a_{\mathrm{f}} / R_{\mathrm{p}}} x^{11 / 2} \mathrm{~d} x}{\int_{2}^{a_{\mathrm{f}} / R_{\mathrm{p}}} \frac{x^{11 / 2}}{1+\frac{19}{22} x^{-2}+\frac{380}{459} x^{-4}+\frac{455}{584} x^{-6}+\frac{133}{165} x^{-8}} \mathrm{~d} x}
$$

and is shown as a function of the final separation in Fig. 8. Note that in Fig. 8, the contribution of the secondary is included in the numerical integration of (33) although it is not explicitly given in (36) above. Evolution from a close initial separation of $2 R_{\mathrm{p}}$ to a wide separation of $10 R_{\mathrm{p}}$ results in only a $\sim 1 \%$ increase in $\mu Q$ over the classical value for all size ratios. Thus, the basic $\ell=2$ tidal mechanism is sufficient for well-separated binaries. On the other hand, if the final separation is smaller, as is the case for most near-Earth binaries, the correction is larger, increasing to $5 \%$ for evolution from $2 R_{\mathrm{p}}$ to $5 R_{\mathrm{p}}$ and $15 \%$ for evolution from $2 R_{\mathrm{p}}$ to $3 R_{\mathrm{p}}$. When making a coarse estimate of the material properties of the system, taking the close orbit into account is not of paramount importance; classical tides will easily provide an order-of-magnitude estimate of $\mu Q$ for even the closest of binary asteroids, though the 
Fig. 8 In terms of the ratio $\mu Q_{\ell=6} / \mu Q_{\ell=2}$ for tidal evolution from $2 R_{\mathrm{p}}$ with both components contributing, systems with $R_{\mathrm{S}} / R_{\mathrm{p}}=0,1$ or a synchronized secondary share the solid curve and are most affected by the close-orbit correction; a system with $R_{\mathrm{S}} / R_{\mathrm{p}}=0.53$ (dashed curve) is the least affected by the close-orbit correction. Overall, the close-orbit correction is roughly $25 \%$ at $2 R_{\mathrm{p}}$ and quickly falls off to $5 \%$ for evolution from $2 R_{\mathrm{p}}$ to $5 R_{\mathrm{p}}$ and to $1 \%$ for evolution to $10 R_{\mathrm{p}}$ for all size ratios. The components are assumed to have similar $\mu Q$ parameters and contribute in an additive sense in (33)

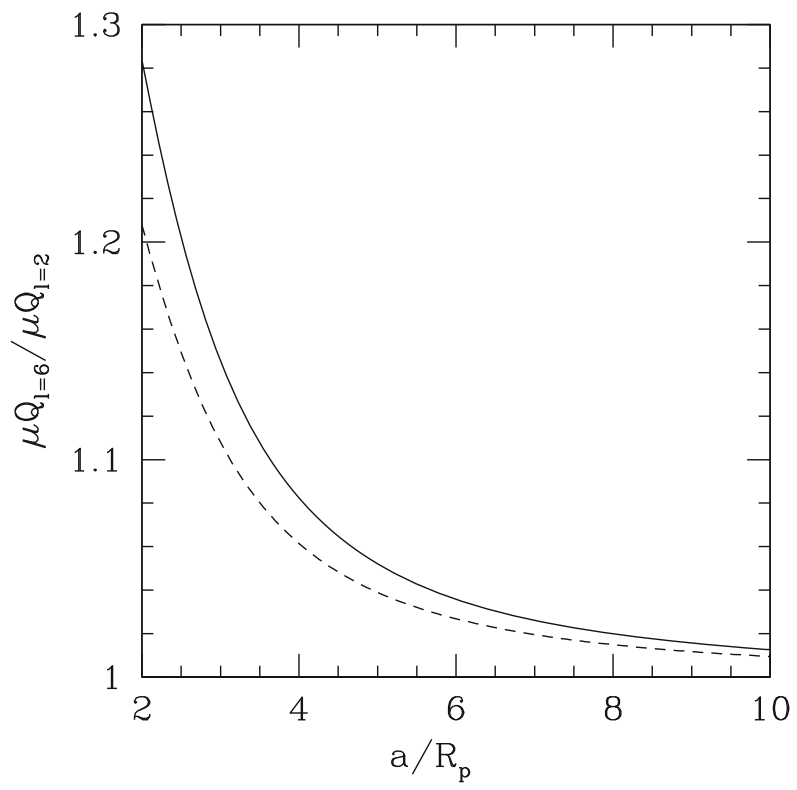

result will be slightly underestimated. Complementarily, if higher-order terms are included and $\mu Q$ is held fixed, the age of the binary must decrease by the same factor as in Fig. 8 meaning that $\ell=2$ tides provide an upper bound on ages for systems with a given $\mu Q$ value.

The use of higher-order terms up to $\ell=6$ is sufficient for exploring the tidal evolution of binary systems with separations greater than $2 R_{\mathrm{p}}$. Additional terms with $\ell>6$ make inconsequential changes to tidal evolution at these separations as illustrated by the rapid fall-off of the contributions of the higher-order terms beyond $2 R_{\mathrm{p}}$ in Table 2 . Moreover, terms with $\ell>6$ leave Figs. $4-8$ unchanged, only having an effect within $2 R_{\mathrm{p}}$. Thus, if one wishes to proceed inward of $2 R_{\mathrm{p}}$, simply using orders of up to $\ell=6$ is insufficient as higher-order terms gain importance the closer one proceeds to the primary. Though we stated earlier that the number of terms required can rapidly become unwieldy, one can approximate their strength. For an arbitrary order $\ell>2$, the term within the square brackets of (33) is approximately

$$
0.8\left(\frac{a}{R_{\mathrm{p}}}\right)^{-2(\ell-2)}\left(\operatorname{sign}\left(\omega_{\mathrm{p}}-n\right)+\left(\frac{R_{\mathrm{s}}}{R_{\mathrm{p}}}\right)^{2 \ell-3} \frac{\mu_{\mathrm{p}} Q_{\mathrm{p}}}{\mu_{\mathrm{s}} Q_{\mathrm{s}}} \operatorname{sign}\left(\omega_{\mathrm{s}}-n\right)\right),
$$

allowing additional terms to be included without explicit calculation of the Love numbers $k_{\ell, \mathrm{p}}$ or manipulation of the Legendre polynomials. Similar terms follow for the changes in spin rates. One must keep in mind that the approximation in (37) is only valid so long as the small angle approximation holds $\left(\sin m \delta \simeq m \delta \propto Q^{-1}\right)$ with $m \leq \ell$, which requires $Q>10$ to retain $1 \%$ accuracy at $m=6$ and larger $Q$ as $m$ increases $^{13}$ (e.g., $Q>20$ for $m=10$ ). Also, having separations of less than $2 R_{\mathrm{p}}$ requires smaller secondaries, since contact occurs at a separation of $\left(1+R_{\mathrm{S}} / R_{\mathrm{p}}\right) R_{\mathrm{p}}$, which reduces the contribution of the secondary due to dependencies upon the size ratio, in addition to demanding consideration of the Roche limit for the system (see Sect. 3).

13 We have applied (21) to estimate the value of $Q$ required. In general, the small angle approximation holds to within $1 \%$ for $Q_{\ell m p q} \sim 4$ or greater. 


\section{Comparison to measurement errors}

Take, for example, 66391 (1999 KW4), the best-studied of the near-Earth binary systems (Ostro et al. 2006; Scheeres et al. 2006). Even with exhaustive analysis of radar imagery, production of three-dimensional shape models of both components, and investigation of the system dynamics, physical parameters of the system are not known with extreme precision. The densities of the primary and secondary components are known to approximately 12 and $25 \%$, respectively. The uncertainty in the density of the primary alone can cause error of more than $30 \%$ in $\dot{\omega}_{\mathrm{p}}, \dot{\omega}_{\mathrm{s}}$, and $\dot{a} / R_{\mathrm{p}}$ according to (31-33), more than the close-orbit correction causes in Figs. 4 and 5. The higher estimated density of the secondary in the 1999 KW4 system of $2.81 \mathrm{~g} / \mathrm{cm}^{3}$, compared to $1.97 \mathrm{~g} / \mathrm{cm}^{3}$ for the primary, directly affects the mass ratio $\kappa$ applied in the equations of tidal evolution as one typically assumes similar densities for the components. Ignoring the density uncertainties, this difference in component densities alone causes a $43 \%$ change in $\kappa$ that, in turn, affects $\dot{\omega}_{\mathrm{p}}$ by a factor of two and $\dot{\omega}_{\mathrm{s}}$ and $\dot{a} / R_{\mathrm{p}}$ by approximately $40 \%$ as well, again, a larger effect than the close-orbit correction to tidal evolution. The calculated value of $\mu Q$ in (35) is affected by density and mass ratio uncertainties in the same way as $\dot{a} / R_{\mathrm{p}}$. Furthermore, uncertainties in densities and the dependence of the mass ratio $\kappa$ on density differences between the components apply at all separations unlike the close-orbit correction, which falls off quickly with increasing separation.

One must also consider the effect of the initial separation of the components at the onset of tidal evolution, a property that is not known for individual systems, but can be estimated from simulations of binary formation mechanisms [e.g., Walsh and Richardson (2006), Walsh et al. (2008)] and given a lower bound by the contact limit at $\left(1+R_{\mathrm{s}} / R_{\mathrm{p}}\right) R_{\mathrm{p}}$. Assuming evolution over the same timescale, if the system had an initial separation $a_{\mathrm{i}}$ instead of $2 R_{\mathrm{p}}$, the effect on $\mu Q$ calculated with classical $\ell=2$ tides raised only on the primary is

$$
\frac{\mu Q_{\mathrm{i}}}{\mu Q_{2}}=\frac{1-\left(\frac{2}{a_{\mathrm{f}} / R_{\mathrm{p}}}\right)^{13 / 2}}{1-\left(a_{\mathrm{i}} / a_{\mathrm{f}}\right)^{13 / 2}} .
$$

For a final separation $a_{\mathrm{f}}$ from $3 R_{\mathrm{p}}$ to $10 R_{\mathrm{p}}$, unless the actual initial separation $a_{\mathrm{i}}$ is within $10 \%$ of the final separation $\left(>0.9 a_{\mathrm{f}}\right)$, the value of $\mu Q$ is affected by less than a factor of two by assuming an initial separation of $2 R_{\mathrm{p}}$. Using up to $\ell=6$ and allowing for tides raised on the secondary with any size ratio do not cause a significant difference in this result.

A similar result is found for the dependence on the final separation of the components, which one typically takes to be the current separation. If $a_{\mathrm{f}}$ is the final (current) separation, then changing the separation to $a_{\mathrm{f}^{\prime}}$ due to, say, a measurement error causes the calculated $\mu Q$ value for tidal evolution from $2 R_{\mathrm{p}}$ to change as

$$
\frac{\mu Q_{\mathrm{f}^{\prime}}}{\mu Q_{\mathrm{f}}}=\frac{1-\left(\frac{2}{a_{\mathrm{f}} / R_{\mathrm{p}}}\right)^{13 / 2}}{\left(a_{\mathrm{f}^{\prime}} / a_{\mathrm{f}}\right)^{13 / 2}-\left(\frac{2}{a_{\mathrm{f}} / R_{\mathrm{p}}}\right)^{13 / 2}} .
$$

We find $\mu Q$ is affected by less than a factor of two if the final (current) separation is known within $10 \%$. From the dependence on the initial and final separations, it is clear that the tidal evolution near the final separation dominates over the early evolution where the close-orbit correction is necessary. In fact, if instead of calculating $\mu Q$, one considers the time taken to tidally evolve to a final separation $a_{\mathrm{f}} \geq 4 R_{\mathrm{p}}$ (by assuming a value of $\mu Q$ instead of an age), the evolution of the separation from $0.9 a_{\mathrm{f}}$ to $a_{\mathrm{f}}$ takes roughly the same amount of time as the evolution from $a_{\mathrm{i}} \leq 2 R_{\mathrm{p}}$ to $0.9 a_{\mathrm{f}}$. Thus, precisely when the close-orbit correction is most 
prominent is also when the system requires the least amount of time to evolve, which causes the mild effect of the close-orbit correction found in Figs. 7 and 8.

Returning to a concrete example, for the $1999 \mathrm{KW} 4$ system, using the equivalent spherical radius of the primary shape model, the separation of the components $a / R_{\mathrm{p}}$ is known to $3 \%$ as $3.87 \pm 0.12$ (Ostro et al. 2006). By (39), this small uncertainty can result in a roughly $20 \%$ error in the calculated $\mu Q$, more than twice the effect of the close-orbit correction in Fig. 8 at $3.87 R_{\mathrm{p}}$. Together with the dependence of the $\mu Q$ calculation on the density values for the components, the accuracy of measurements of physical parameters in the 1999 KW4 system is more important than accounting for the proximity of the components to one another.

\section{Discussion}

We have derived the equations of tidal evolution to arbitrary order in the Legendre polynomial expansion of the separation between two spherical bodies in a circular and equatorial mutual orbit allowing for accurate representation of evolution within five primary radii. Equations written in terms of the Love number $k_{\ell}$ are applicable to any binary system, while equations where the Love number has been evaluated have assumed the bodies involved have rigidities that dominate their self-gravitational stress (characteristic of bodies less than roughly $200 \mathrm{~km}$ in radius). Because higher-order terms cause tidal evolution to proceed faster, choosing to ignore them produces upper limits on tidal evolution timescales and lower limits on material properties in terms of the product of rigidity and the tidal dissipation function. However, we have shown that the correction for close orbits has only a minor integrated effect on outward tidal evolution and the calculation of material properties, comparable to or less than the effect of uncertainties in measurable properties such as density, mass ratio, and semimajor axis (scaled to the radius of the primary component). In the case of outward evolution, the binary system evolves rapidly through the range of separations where the close-orbit correction is strongest, so one can safely ignore the correction to obtain order-of-magnitude estimates of timescales and material properties using the classical equations for tidal evolution. Accounting for higher orders is more applicable to studying, famously in the case of Phobos, observed secular accelerations and the infall of a secondary to the surface of its primary where the higher-order terms instead gain strength.

Though we have presented the expansion of the gravitational potential and the resulting equations of tidal evolution in the context of two asteroids in mutual orbit, the essence of this work could be generalized for use in the determination of the Roche limit and the study of close flybys. The use of a higher-order expansion of the gravitational potential in terms of Legendre polynomials is warranted whenever the separation of two bodies is within five times the radius of one of the bodies ${ }^{14}$ (see Table 1). Historically, in the context of disruption of a body at the Roche limit or due to a close flyby of a larger body (Sridhar and Tremaine 1992; Richardson et al. 1998; Holsapple and Michel 2006, 2008; Sharma et al. 2006; Sharma 2009), stresses are only considered in the much smaller secondary while the primary is assumed to be rigid. For small secondaries, the cohesionless Roche limit of $1.5-2 R_{\mathrm{p}}$ is much larger than $5 R_{\mathrm{S}}$ such that higher-order terms in the potential expansion are not necessary. However, as larger secondaries are considered $\left(R_{\mathrm{S}} / R_{\mathrm{p}}>0.1\right)$, higher-order terms in the gravitational potential will further stress the secondary near the Roche limit. Also, with components of increasingly similar size, the assumption of a rigid primary is not appropriate; the tidal stress

14 The potential felt by the primary requires higher-order terms with $\ell>2$ if the separation is less than $5 R_{\mathrm{p}}$; the potential felt by the secondary requires higher-order terms with $\ell>2$ if the separation is less than $5 R_{\mathrm{S}}$. 
on the primary will deform it from a spherical shape and produce an external potential as in Sect. 4 that will in turn further stress the secondary. If the components are not spin-locked, tidal torques will also play a role in stressing the secondary. Thus, if evaluating the Roche limit for components of similar size and/or components that are not spin-locked, one must consider the description presented here. For disruption during a close flyby, or simply modification of the spin state of the passing body (Scheeres 2001; Scheeres et al. 2000, 2004), one must consider the proximity of the flyby in terms of the expansion of the gravitational potential and whether or not tidal bulges can be raised on the components that would produce torques capable of further altering the spin state of either component.

It is also important to remember that the higher-order theory presented here has implicitly assumed initially spherical bodies. Extension of this work from spheres to ellipsoids or to arbitrary shapes would affect the mutual gravitational potential, linear and angular momentum balance, and orbital equations as described by Scheeres (2009) and Sharma (2010). Once the shape is made nonspherical in the absence of a tidal potential, the system is subject to a "direct" torque that naturally occurs from the changing gravitational pull felt by the orbiting component due to the nonspherical shape of the other component. Accounting for the tidal potential introduces the "indirect" torque described here due to the deformation of one component by the gravitational presence of the other component. Because the amplitude of the tidal bulge on asteroids, the parameter $\lambda$ in this work, can be very small due to its direct dependence on the ratio of self-gravitational stress to rigidity, its direct dependence on the mass ratio, and its inverse dependence on the separation raised to the third (or higher) power, natural deviations from a spherical shape may exceed the amplitude of the tidal bulge. However, one must recall that the direct torques due to a nonspherical shape will change direction as the body rotates under the orbiting component tending to cancel the pre- and post-encounter effects of the torque as opposed to the indirect torque that is in a consistent direction so long as the bulge always leads or lags the orbiting component. It may be important to consider direct torques due to natural departures from a spherical shape via the use of shape models: oblate or prolate spheroids, triaxial ellipsoids, or vertex models such as those made for the components of the 1999 KW4 binary system and other asteroids.

Acknowledgments The authors are indebted to the two referees whose detailed reviews and insightful suggestions improved the clarity and quality of the manuscript. The authors are especially grateful to Michael Efroimsky for many discussions on the finer points of tidal theory and Celestial Mechanics. This work was supported by NASA Planetary Astronomy grants NNG04GN31G and NNX07AK68G to Jean-Luc Margot.

Open Access This article is distributed under the terms of the Creative Commons Attribution Noncommercial License which permits any noncommercial use, distribution, and reproduction in any medium, provided the original author(s) and source are credited.

\section{References}

A'Hearn, M.F., Belton, M.J.S., Delamere, W.A., Kissel, J., Klaasen, K.P., McFadden, L.A., Meech, K.J., Melosh, H.J., Schultz, P.H., Sunshine, J.M., Thomas, P.C., Veverka, J., Yeomans, D.K., Baca, M.W., Busko, I., Crockett, C.J., Collins, S.M., Desnoyer, M., Eberhardy, C.A., Ernst, C.M., Farnham, T.L., Feaga, L., Groussin, O., Hampton, D., Ipatov, S.I., Li, J., Lindler, D., Lisse, C.M., Mastrodemos, N., Owen, W.M., Richardson, J.E., Wellnitz, D.D., White, R.L.: Deep impact: Excavating comet tempel 1. Science 310, 258-264 (2005)

Behrend, R., Bernasconi, L., Roy, R., Klotz, A., Colas, F., Antonini, P., Aoun, R., Augustesen, K., Barbotin, E., Berger, N., Berrouachdi, H., Brochard, E., Cazenave, A., Cavadore, C., Coloma, J., Cotrez, V., Deconihout, S., Demeautis, C., Dorseuil, J., Dubos, G., Durkee, R., Frappa, E., Hormuth, F., Itkonen, T., Jacques, C., Kurtze, L., Laffont, A., Lavayssière, M., Lecacheux, J., Leroy, A., Manzini, F., Masi, G., Matter, 
D., Michelsen, R., Nomen, J., Oksanen, A., Pääkkönen, P., Peyrot, A., Pimentel, E., Pray, D., Rinner, C., Sanchez, S., Sonnenberg, K., Sposetti, S., Starkey, D., Stoss, R., Teng, J.P., Vignand, M., Waelchli, N.: Four new binary minor planets: (854) Frostia, (1089) Tama, (1313) Berna, (4492) Debussy. Astron. Astroph. 446, 1177-1184 (2006)

Bills, B.G., Neumann, G.A., Smith, D.E., Zuber, M.T.: Improved estimate of tidal dissipation within Mars from MOLA observations of the shadow of Phobos. J. Geophys. Res. 110, 2376-2406 (2005)

Burns, J.A.: Elementary derivation of the perturbation equations of Celestial Mechanics. Am. J. Phys. 44, 944949 (1976)

Burns, J.A.: Orbital evolution. In: Burns, J.A. (ed.) Planetary Satellites, pp. 113-156. University of Arizona Press, Tucson (1977)

Chandrasekhar, S.: Ellipsoidal Figures of Equilibrium. Yale University Press, New Haven (1969)

Danby, J.M.A.: Fundamentals of Celestial Mechanics. Willman-Bell, Richmond (1992)

Darwin, G.H.: On the bodily tides of viscous and semi-elastic spheroids, and on the ocean tides upon a yielding nucleus. Philos. Trans. R. Soc. London 170, 1-35 (1879a)

Darwin, G.H.: On the precession of a viscous spheroid, and on the remote history of the Earth. Philos. Trans. R. Soc. London 170, 447-538 (1879b)

Darwin, G.H.: On the secular changes in the elements of the orbit of a satellite revolving about a tidally distorted planet. Philos. Trans. R. Soc. London 171, 713-891 (1880)

Descamps, P., Marchis, F.: Angular momentum of binary asteroids: Implications for their possible origin. Icarus 193, 74-84 (2008)

Descamps, P., Marchis, F., Michalowski, T., Vachier, F., Colas, F., Berthier, J., Assafin, M., Dunckel, P.B., Polinska, M., Pych, W., Hestroffer, D., Miller, K.P.M., Vieira-Martins, R., Birlan, M., Teng-Chuen-Yu, J.P., Peyrot, A., Payet, B., Dorseuil, J., Léonie, Y., Dijoux, T.: Figure of the double asteroid 90 Antiope from adaptive optics and lightcurve observations. Icarus 187, 482-499 (2007)

Efroimsky, M., Williams, J.G.: Tidal torques: A critical review of some techniques. Celest. Mech. Dyn. Astron. 104, 257-289 (2009)

Ferraz-Mello, S., Rodríguez, A., Hussmann, H.: Tidal friction in close-in satellites and exoplanets: The Darwin theory re-visited. Celest. Mech. Dyn. Astron. 101, 171-201 (2008)

Gerstenkorn, H.: Über Gezeitenreibung beim Zweikörperproblem. Zeitschrift Fur Astrophysik 36, 245274 (1955)

Goldreich, P.: On the eccentricity of satellite orbits in the solar system. Mon. Not. R. Astron. Soc. 126, 257268 (1963)

Goldreich, P.: History of the lunar orbit. Rev. Geophys. Space Phys. 4, 411-439 (1966)

Goldreich, P., Sari, R.: Tidal evolution of rubble piles. Astroph. J. 691, 54-60 (2009)

Goldreich, P., Soter, S.: Q in the solar system. Icarus 5, 375-389 (1966)

Holsapple, K.A., Michel, P.: Tidal disruptions: A continuum theory for solid bodies. Icarus 183, 331-348 (2006)

Holsapple, K.A., Michel, P.: Tidal disruptions. II. A continuum theory for solid bodies with strength, with applications to the Solar System. Icarus 193, 283-301 (2008)

Kaula, W.M.: Tidal dissipation by solid friction and the resulting orbital evolution. Rev. Geophys. 2, 661685 (1964)

Lambeck, K.: On the orbital evolution of the Martian satellites. J. Geophys. Res. 84, 5651-5658 (1979)

Love, A.E.H.: A Treatise on the Mathematical Theory of Elasticity. Dover, New York (1927)

MacDonald, G.J.F.: Tidal friction. Rev. Geophys. Space Phys. 2, 467-541 (1964)

MacRobert, T.M.: Spherical Harmonics. Pergamon Press, Oxford (1967)

Marchis, F., Descamps, P., Berthier, J., Hestroffer, D., Vachier, F., Baek, M., Harris, A.W., Nesvorný, D.: Main belt binary asteroidal systems with eccentric mutual orbits. Icarus 195, 295-316 (2008a)

Marchis, F., Descamps, P., Baek, M., Harris, A.W., Kaasalainen, M., Berthier, J., Hestroffer, D., Vachier, F.: Main belt binary asteroidal systems with circular mutual orbits. Icarus 196, 97-118 (2008b)

Margot, J.L., Nolan, M.C., Benner, L.A.M., Ostro, S.J., Jurgens, R.F., Giorgini, J.D., Slade, M.A., Campbell, D.B.: Binary asteroids in the near-earth object population. Science 296, 1445-1448 (2002)

Margot, J.L., Nolan, M.C., Negron, V., Hine, A.A., Campbell, D.B., Howell, E.S., Benner, L.A.M., Ostro, S.J., Giorgini, J.D., Marsden, B.G.: 1937 UB (Hermes). IAU Circ. 8227, 2 (2003)

Margot, J.L., Pravec, P., Nolan, M.C., Howell, E.S., Benner, L.A.M., Giorgini, J.D., F., J.R., Ostro, S.J., Slade, M.A., Magri, C., Taylor, P.A., Nicholson, P.D., Campbell, D.B.: Hermes as an exceptional case among binary near-earth asteroids. In: IAU General Assembly (2006)

Merline, W.J., Close, L.M., Shelton, J.C., Dumas, C., Menard, F., Chapman, C.R., Slater, D.C., Keck Telescope, W.M. II.: Satellites of minor planets. IAU Circ. 7503, 3 (2000)

Michałowski, T., Bartczak, P., Velichko, F.P., Kryszczyńska, A., Kwiatkowski, T., Breiter, S., Colas, F., Fauvaud, S., Marciniak, A., Michałowski, J., Hirsch, R., Behrend, R., Bernasconi, L., Rinner, C., Charbonnel, S.: Eclipsing binary asteroid 90 Antiope. Astron. Astroph. 423, 1159-1168 (2004) 
Mignard, F.: The evolution of the lunar orbit revisited. I. Moon and Planets 20, 301-315 (1979)

Mignard, F.: The evolution of the lunar orbit revisited. II. Moon and Planets 23, 185-201 (1980)

Mignard, F.: The lunar orbit revisited. III. Moon Planets 24, 189-207 (1981)

Munk, W.H., MacDonald, G.J.F.: The Rotation of the Earth. Cambridge University Press, Cambridge (1960)

Murray, C.D., Dermott, S.F.: Solar System Dynamics. Cambridge University Press, Cambridge (1999)

Ostro, S.J., Margot, J.L., Benner, L.A.M., Giorgini, J.D., Scheeres, D.J., Fahnestock, E.G., Broschart, S.B., Bellerose, J., Nolan, M.C., Magri, C., Pravec, P., Scheirich, P., Rose, R., Jurgens, R.F., De Jong, E.M., Suzuki, S.: Radar imaging of binary near-Earth asteroid (66391) 1999 KW4. Science 314, 12761280 (2006)

Peale, S.J.: Origin and evolution of the natural satellites. Annu. Rev. Astron. Astrophys. 37, 533-602 (1999)

Pravec, P., Harris, A.W.: Binary asteroid population 1. Angular momentum content. Icarus 190, 250-259 (2007)

Pravec, P., Kusnirak, P., Warner, B., Behrend, R., Harris, A.W., Oksanen, A., Higgins, D., Roy, R., Rinner, C., Demeautis, C., Abbeel, F.van den, Klotz, A., Waelchli, N., Alderweireldt, T., Cotrez, V., Brunetto, L.: 1937 UB (Hermes). IAU Circ. 8233, 3 (2003)

Redmond, J.C., Fish, F.F.: The luni-tidal interval in Mars and the secular acceleration of Phobos. Icarus 3, 87-91 (1964)

Richardson, D.C., Bottke, W.F., Love, S.G.: Tidal distortion and disruption of Earth-crossing asteroids. Icarus 134, 47-76 (1998)

Richardson, D.C., Walsh, K.J.: Binary minor planets. Annu. Rev. Earth Planet Sci. 34, 47-81 (2006)

Richardson, J.E., Melosh, H.J., Lisse, C.M., Carcich, B.: A ballistics analysis of the Deep Impact ejecta plume: Determining comet Tempel 1's gravity, mass, and density. Icarus 190, 357-390 (2007)

Rubincam, D.P.: The early history of the lunar inclination. NASA-GSFC Rep. X-592-73-328, Goddard Space Flight Center, Greenbelt, Md. (1973)

Rubincam, D.P.: Radiative spin-up and spin-down of small asteroids. Icarus 148, 2-11 (2000)

Scheeres, D.J.: Changes in rotational angular momentum due to gravitational interactions between two finite bodies. Celest. Mech. Dyn. Astron. 81, 39-44 (2001)

Scheeres, D.J.: Stability of the planar full 2-body problem. Celest. Mech. Dyn. Astron. 104, 103-128 (2009)

Scheeres, D.J., Ostro, S.J., Werner, R.A., Asphaug, E., Hudson, R.S.: Effects of gravitational interactions on asteroid spin states. Icarus 147, 106-118 (2000)

Scheeres, D.J., Marzari, F., Rossi, A.: Evolution of NEO rotation rates due to close encounters with Earth and Venus. Icarus 170, 312-323 (2004)

Scheeres, D.J., Fahnestock, E.G., Ostro, S.J., Margot, J.L., Benner, L.A.M., Broschart, S.B., Bellerose, J., Giorgini, J.D., Nolan, M.C., Magri, C., Pravec, P., Scheirich, P., Rose, R., Jurgens, R.F., De Jong, E.M., Suzuki, S.: Dynamical configuration of binary near-Earth asteroid (66391) 1999 KW4. Science 314, 1280-1283 (2006)

Schellart, W.P.: Shear test results for cohesion and friction coefficients for different granular materials: Scaling implications for their usage in analogue modelling. Tectonophys 324, 1-16 (2000)

Sharma, I.: The equilibrium of rubble-pile satellites: The Darwin and Roche ellipsoids for gravitationally held granular aggregates. Icarus 200, 636-654 (2009)

Sharma, I.: Equilibrium shapes of rubble-pile binaries: The Darwin ellipsoids for gravitationally held granular aggregates. Icarus 205, 638-657 (2010)

Sharma, I., Jenkins, J.T., Burns, J.A.: Tidal encounters of ellipsoidal granular asteroids with planets. Icarus 183, 312-330 (2006)

Smith, J.C., Born, G.H.: Secular acceleration of Phobos and Q of Mars. Icarus 27, 51-53 (1976)

Sridhar, S., Tremaine, S.: Tidal disruption of viscous bodies. Icarus 95, 86-99 (1992)

Szeto, A.M.K.: Orbital evolution and origin of the martian satellites. Icarus 55, 133-168 (1983)

Taylor, P.A., Margot, J.L.: Tidal evolution of solar system binaries. Bull. Am. Astron. Soc. 39, 439 (2007)

Taylor, P.A., Margot, J.L.: Binary asteroid systems: Tidal end states and estimates of material properties. (2010, submitted)

Vokrouhlický, D., Čapek, D.: YORP-induced long-term evolution of the spin state of small asteroids and meteoroids: Rubincam's approximation. Icarus 159, 449-467 (2002)

Walsh, K.J., Richardson, D.C.: Binary near-Earth asteroid formation: Rubble pile model of tidal disruptions. Icarus 180, 201-216 (2006)

Walsh, K.J., Richardson, D.C., Michel, P.: Rotational breakup as the origin of small binary asteroids. Nature 454, 188-191 (2008)

Weidenschilling, S.J., Paolicchi, P., Zappalà, V.: Do asteroids have satellites? In: Binzel, R.P., Gehrels, T., Matthews, M.S. (eds.) Asteroids II, pp. 643-660. University of Arizona Press, Tucson (1989) 